\title{
Silicon crystals for steering high-intensity particle beams at ultrahigh-energy accelerators
}

\author{
A. Mazzolari, ${ }^{1}$ M. Romagnoni, ${ }^{1,2}$ E. Bagli, ${ }^{1}$ L. Bandiera, ${ }^{1}$ S. Baricordi, ${ }^{3}$ R. Camattari, ${ }^{1}$ D. Casotti, ${ }^{1,2}$ M. Tamisari,,${ }^{1,4}$ \\ A. Sytov, ${ }^{1}$ V. Guidi, ${ }^{1,2}$ G. Cavoto, ${ }^{5,6}$ S. M. Carturan, ${ }^{7,8}$ D. De Salvador,${ }^{7,8}$ A. Balbo, ${ }^{9}$ G. Cruciani, ${ }^{2}$ Thu Nhi Tran Caliste, ${ }^{10}$ \\ R. Verbeni, ${ }^{10}$ N. Pastrone, ${ }^{11}$ L. Lanzoni, ${ }^{12}$ A. Rossall, ${ }^{13}$ J. A. van den Berg, ${ }^{13}$ R. Jenkins, ${ }^{14}$ and P. Dumas ${ }^{14}$ \\ ${ }^{1}$ INFN Sezione di Ferrara, Via Saragat 1, 44122 Ferrara, Italy \\ ${ }^{2}$ Università degli Studi di Ferrara, Dipartimento di Fisica e Scienze della Terra, Via Saragat 1/C, 44122 Ferrara, Italy \\ ${ }^{3}$ Dott. S. Baricordi, Via O. Putinati 67, 44123, Ferrara, Italy \\ ${ }^{4}$ Dipartimento di Scienze Biomediche e Chirurgico specialistiche Università di Ferrara \\ ${ }^{5}$ Università degli Studi di Roma La Sapienza, Piazzale Aldo Moro 2, 00185 Rome, Italy \\ ${ }^{6}$ INFN Sezione di Roma, Piazzale Aldo Moro 2, 00185 Rome, Italy \\ ${ }^{7}$ INFN Laboratori Nazionali di Legnaro, Viale dell'Università 2, 35020 Legnaro (Padova), Italy \\ ${ }^{8}$ Dipartimento di Fisica e Astronomia, Università degli Studi di Padova, Via Marzolo 8, 35131 Padova, Italy \\ ${ }^{9}$ Centro di Studi sulla Corrosione e Metallurgia “Aldo Daccò”, Dipartimento di Ingegneria, Università di Ferrara, \\ Via Saragat 4a, 44122 Ferrara, Italy \\ ${ }^{10}$ European Synchrotron Radiation Facility (ESRF), 71 avenue des Martyrs, 38000 Grenoble, France \\ ${ }^{11}$ INFN Sezione di Torino, Via Giuria 1, 10125 Torino, Italy \\ ${ }^{12}$ Dipartimento di Ingegneria "Enzo Ferrari”, Università degli Studi di Modena e Reggio Emilia Via Pietro Vivarelli 10, 41125 Modena, Italy \\ ${ }^{13}$ Ion Beam Centre, School of Computing and Engineering, University of Huddersfield, Huddersfield, HD1 3DH, United Kingdom \\ ${ }^{14}$ QED Technologies North America, 1040 University Avenue Rochester, New York 14607, USA
}

(Received 28 June 2020; revised 9 July 2020; accepted 23 November 2020; published 3 February 2021)

\begin{abstract}
Experimental results and simulation models show that crystals might play a relevant role for the development of new generations of high-energy and high-intensity particle accelerators and might disclose innovative possibilities at existing ones. In this paper we describe the most advanced manufacturing techniques of crystals suitable for operations at ultrahigh energy and ultrahigh intensity particle accelerators, reporting as an example of potential application the collimation of the particle beams circulating in the Large Hadron Collider at CERN, which will be upgraded through the addition of bent crystals in the frame of the High Luminosity Large Hadron Collider project.
\end{abstract}

DOI: 10.1103/PhysRevResearch.3.013108

\section{INTRODUCTION}

In a crystalline lattice atoms occupy well defined positions, resulting in regularly spaced distributions of electron and nuclei densities. This structure can be described as made by planes and rows of atoms. Exploiting this property, coherent scattering of electromagnetic radiation (e.g., x rays) or of massive particle beams (e.g., neutrons, electrons, protons, etc.) by a crystal lattice occurs when properly mutually oriented.

Since the early 1980s, bent crystals started to find application in accelerators as elements useful for beam steering [1-7] or splitting [8]. This was possible thanks to the availability of innovative ideas $[7,9,10]$ and silicon crystals of sufficiently high crystalline perfection. Indeed, as a charged particle impinges on atomic planes or axis of a crystal at a sufficiently small angle, it is captured under a channeling

Published by the American Physical Society under the terms of the Creative Commons Attribution 4.0 International license. Further distribution of this work must maintain attribution to the author(s) and the published article's title, journal citation, and DOI. regime [11]. As the crystal is bent, its atomic planes become a pathway for propagation of the particle inside the crystal and then the deflection of the particle beam occurs [5]. A first generation of experiments conducted in the 1980s recorded deflection efficiencies in the order of few percent or less, which was raised to few tens of percent in the 1990s [5] mainly thanks to a proper design of the crystal geometry [12] and innovative crystal manufacturing approaches [13]. In more recent years, the discovery of numerous effects appearing whenever the crystal planes [14-18] or the crystal axes [19-24] are aligned to the charged particle beam direction was the result of reliable physical models, innovative crystal bending schemes $[25,26]$, and considerable improvements in crystal manufacturing techniques $[13,27]$. In particular, in a pioneering experiment conducted at the Institute for High Energy Physics (Protvino, Moscow), a bent crystal installed in the ring as a primary element upstream of a collimator has reduced the radiation levels downstream in the accelerator by a factor of 2 (see [26] and references therein).

The most recent results achieved at the Large Hadron Collider (LHC) [6,28] and the Super Proton Synchrotron at CERN [29-31] demonstrate that the technology readiness level reached by this technique makes it relevant for efficient 
deflection of particle beams in ultrahigh-energy and intensity accelerators. Indeed, after a long R\&D mainly carried in the frame of the UA9 collaboration, the use of bent crystals have been recently added as a part of the baseline upgrade of the collimation system of the LHC in the frame of the High Luminosity Large Hadron Collider (HL-LHC) project [32,33] at CERN.

Bent crystals could be also used to extract the halo of the beam circulating in the LHC toward an extracted line with no cost for the collider-mode experiments, enabling a ground-breaking physics program accessible within fixedtarget experiments with the multi-TeV proton and ion beams $[34,35]$ of the LHC. While this proposal would result in a considerable effort, fixed target experiments using existing detectors and smaller changes to the LHC infrastructure have been suggested [36-38] for operations with the $\mathrm{LHCb}$ and ALICE collaborations. Operations with the $\mathrm{LHCb}$ detector are motivated by the aim to measure the electric and magnetic dipole moments of charmed baryons [37,39-44], resulting in the determination of the magnetic moment of the charm quark. Within ALICE the use of bent crystals have been suggested in view of possible fixed-target experiments aiming to investigate heavy-Ion, hadron, spin, and astroparticle physics $[45,46]$.

Crystal-based solutions [47] are also under consideration for the slow extraction of circulating beams in the Super Proton Synchrotron (SPS) at CERN [48] or at synchrotron facilities [49]. Aside from particle beam steering, crystals could also be used as innovative elements for generation of hard radiation [50,51] or positron [52] beams and for the realization of compact photoconverter [8] and forward electromagnetic calorimeters $[53,54]$.

As compared to traditional magnetic optical elements used in accelerators, crystals operate without the need of a power source, generally do not require a cryogenic environment, and are extremely compact and lightweight, typically less than $1 \mathrm{~kg}$, making them appealing for integration in particle accelerators. The use of crystals have been suggested also for the Future Circular Collider (FCC) [39,41,55-57], the International Linear Collider (ILC) [52,58], the Compact Linear Collider (CLIC) [58,59], and the Circular Electron Positron Collider (CEPC) [60] and it could be exploited in a future muon collider design $[61,62]$. Given the extremely high intensity and energy reached in modern and future particle accelerators such as the HL-LHC or in future planned ones, the implementation of bent crystals at these facilities demands facing various technological challenges and a considerable improvement of the state-of-the-art in the manufacturing of crystals for scientific or technological application.

As an example of potential application, in this paper we focus our attention mainly on the solutions for manufacturing and characterization of crystals with a geometry optimized for the collimation of the LHC ion beam in the framework of the HL-LHC project. The successful development of such crystals is based on a merging of ultramodern technologies used in microelectronics, x-ray science, ultraprecise optical and mechanical machining, and might open innovative schemes for particle beam deflection at future accelerators. For example, the geometry of crystals suitable for collimation $[63,64]$ or extraction $[37,38]$ of the LHC circulating beams are similar, so a revisitation of the crystals developed for the collimation of the LHC beam might enlarge the possibilities for fixed-target experiments at the $\mathrm{LHC}$.

\section{BENT CRYSTALS AS PARTICLE BEAM COLLIMATORS}

A reliable collimation system is a key component of any particle accelerator, especially for ultrahigh energy/intensity colliders based on a superconducting magnet. In particle accelerators, indeed, various mechanisms can cause particles of the beam to enter into unstable orbits, causing the formation of a beam halo and the growth of the beam emittance. This turns into uncontrolled beam losses, potentially causing damage to the accelerator or other perturbations to the operation such as activation of the accelerator components, increased background to the experiments, the quenching of superconducting magnets, diffuse radiation damage, etc. The main purpose of a collimation system is to safely dispose of beam halo losses: a classical solution to this problem consists of the adoption of a multistage collimation system, where a sequence of targets intercepts the halo of the beam. The system effectiveness is determined by the interaction between the beam particles and the collimator active material, i.e., the electromagnetic, elastic, and inelastic nuclear interactions. While elastic interactions do not change the structure of the target or of the particle, inelastic interactions may result in multiparticle final states (with the relevant case of single or double diffractive events). These reactions may result in deleterious effects to the accelerator components, such as targets, detectors, collimators, and the general accelerator environment, and could represent a relevant issue especially for accelerators such as the HL-LHC or the FCC, where extremely high beam intensities and energies are foreseen. The concern related to quenches of cold magnets is particularly important for the LHC and determines the most challenging requirement for the LHC collimation system, which demands a cleaning efficiency larger than $99.9 \%[65,66]$.

In the present LHC collimation scheme (see Fig. 1), a "primary collimator" intercepts the beam halo. A fraction of particles is absorbed in the collimator, while the remaining produces a "secondary halo" and a spray of unwanted "new" particles emerging as a result of single or double diffractive collision events. The particles that escaped from the primary collimator are partially intercepted by secondary collimators, exhibiting a similar behavior to the primary one. If needed, additional collimators are installed after the secondary one. The overall result of this collimation chain is to absorb the beam halo and the method constitutes a classical solution at various accelerators [67-71]. This approach might not provide sufficient cleaning in operations with future accelerators, such as the HL-LHC at CERN, where the stored energy will approach $700 \mathrm{MJ}$ per beam [32,72]. In particular, for the case of operations with heavy ions (partially stripped or not), the collimation efficiency degrades [73] as a consequence of the large cross section of fragmentation processes, resulting in isotopes of different rigidities. Often these ions do not receive a sufficiently large angular kick to be intercepted by the secondary collimators and they deviate from the main beam. Such lost ions could cause a superconducting magnet to quench, or in the worst case, damage the hardware of the accelerator. For 


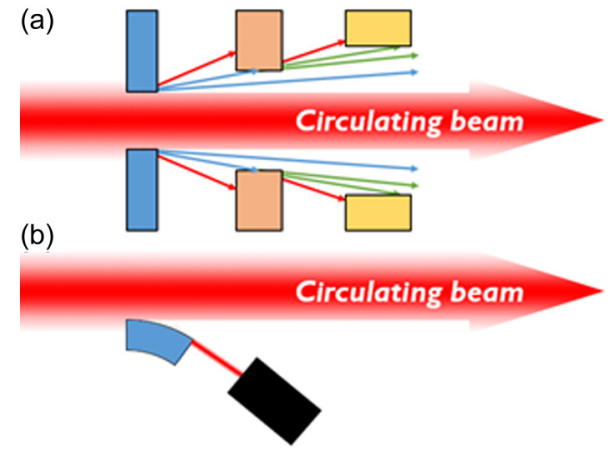

FIG. 1. (a) The classical approach for the collimation of circulating beams in a particle accelerator is based on a chain of materials placed at different apertures along the beam path. Interaction between the "primary halo" and a first block of material (blue box) leads to partial absorption of the halo and, as a by-product of the interaction, to a spray of particles (secondary halo) made primary of halo particles (red arrows) which are not stopped and "new particles" generated in single or double diffractive events (green or blue arrows). The secondary halo is partially absorbed by a second block, which plays the same role for the secondary halo as the first block for the primary halo. (b) Collimation based on a bent crystal as primary collimator. A bent crystal (blue) is aligned to the primary halo and channels its particles between the crystal atomic planes. Channeled particles are efficiently deflected to a massive absorber (black). Given the nature of interaction between the crystal and the beam, the rate of inelastic interactions is strongly suppressed. The elements depicted in this figure have sizes that are not to scale. This figure is inspired by Figs. $5-18$ of Chap. 2 of [75].

the case of the LHC, compared to protons the efficiency of cleaning with heavy ions has been observed to be orders of magnitude worse [74].

The regular distributions of atoms in a crystals lattice make them suitable targets for collimation purposes. These results have been already achieved at RHIC in the collimation of circulating ions beams [76] and have been suggested for the collimation of the LHC in [64] and subsequently studied in more detail in [63]. When the atomic planes of a crystal are not aligned to the high-energy particle beam, interaction between the beam and the crystal is very similar to the interaction that the beam would experience in an amorphous target [77]. As the crystal is aligned to the particle beam in order to excite channeling [5], beam-crystal interactions show unique features. Indeed, under this circumstance each particle of the beam interacts with the electric field naturally present between atomic planes. This interaction drastically influences the motion of each particle of the beam, which passes from a "random" motion for the case of nonalignment, to an oscillatory motion between two neighboring atomic planes for the case of alignment [5]. For example, in case of interaction with a silicon crystal, the wavelength of the oscillation is $\sim 56 \mu \mathrm{m}$ if the channeled particle energy is $400 \mathrm{GeV}$, and rises up to $\sim 235 \mu \mathrm{m}$ at $7 \mathrm{TeV}$. Therefore, particles move across the crystal in regions of low-atomic density. Crystals whose geometry is optimized to maximize beam steering lead an efficiency up to $\sim 83 \%$ for operations in "single-pass" mode $[15,18]$, and up to values close to $100 \%$ thanks to the multipass mechanism [10] in the case of operations in circular machines [12]. Crys- tals robustness against high-energy and high-intensity beams have been assessed under various conditions [5], recently also under conditions similar to the ones manifesting in case of accidental fast irradiation in the LHC [78].

Assuming that a sufficiently robust absorber to dispose the extract beam is available, with respect to the classical collimation schemes, a scheme based on crystals might deliver two potential advantages:

(1) As a crystal is aligned to channel particle beams between its bent atomic planes, the crystal acts as a low-loss waveguide for the beam $[79,80]$, resulting in a strong suppression of fragmentation $[81,82]$ and nuclear interactions with respect to the case of nonalignment and with respect to the case of traditional schemes.

(2) The reduction of the machine electromagnetic impedance. While the classical collimators are meter-long bulky objects and many replica of them are needed, only one single crystal collimator as short as a few $\mathrm{mm}$ can be used, followed by a single - although more massive than standard collimators-absorber (per beam and per collimation plane: horizontal and vertical).

\section{CRYSTAL MANUFACTURING AND CHARACTERIZATION}

The manufacturing of bent crystals and benders for applications in particle accelerators requires a highly multidisciplinary approach. In particular, the merging of different techniques typically used for silicon micromachining was needful, such as ultrahigh precision optical and mechanical machining, the most advanced x-ray characterization techniques, and capabilities in ultraprecise metrology.

In the following we discuss the features of the crystals which might influence steering efficiency in modern particle accelerators, focusing our attention in particular on the manufacturing of crystals that could be suitable for collimation of the HL-LHC proton or ion circulating beams.

\section{A. Starting material: Silicon wafers of ultrahigh crystalline quality}

Crystals are usually manufactured starting from $100 \mathrm{~mm}$ diameter silicon wafers a few $\mathrm{mm}$ thick (typically $2 \mathrm{~mm}$ ). The crystallographic orientation of the largest surfaces of the wafers was (110). The orientation of the largest faces of the wafer determines the orientation of the crystalline planes that will interact with the particle beam. Channeling efficiency and the rate of inelastic interactions [80] are mainly influenced by the distance between atomic planes and their atomic density, as a result the most performing planes are the (110) and the (111) [5]. The average distance between (111) planes $(1.568 \AA)$ is smaller than for (110) planes (1.92 $\AA$ ), consequently the steering efficiency of the latter is higher and the rate of inelastic interaction with the lattice is lower. To confirm this statement, in [80] the performance of crystals with thickness and bending angle suitable for the collimation of the HL-LHC circulating beam were compared in terms of the ratio between the rate of inelastic interactions occurring as the crystals were oriented and not oriented to excite channeling of a $400 \mathrm{GeV}$ 
proton beam. As (110) crystals were oriented for channeling, nuclear interactions reduced to only $\sim 27 \%$ with respect to a condition of nonalignment. For crystals offering (111) channeling planes the reduction leveled to $\sim 36 \%$.

To aid the further manufacturing steps, wafers with "miscut angle" (the angle between the physical surface and atomic planes of the crystal) less than or equal to $200 \mu \mathrm{rad}$ were selected from a large stock of wafers. From this subset, wafers of highest crystalline perfection were selected.

Among the various crystallographic defects that might be present in a crystal, dislocations play the most detrimental role for the channeling efficiency. Presence of this defect results in a deformation field that propagates in the crystal up to large distances from the location where the dislocation appears. A particle that is initially channeled, interacting with the deformation field induced by a dislocation, would suffer "immediate" dechanneling. In order not to degrade the channeling efficiency, a dislocation density lower than $1 / \mathrm{cm}^{2}$ is required [83]. With the aim to satisfy the requirement on what is nowadays considered as an extremely low density of dislocations even for microelectronics (where a level of $50 \mathrm{pits} / \mathrm{cm}^{2}$ is accepted), a large stock of wafers was processed with chemical etchings capable of highlighting the presence of single dislocations $[84,85]$. The wafers that satisfied the requirement of less than one dislocations $/ \mathrm{dm}^{2}$ were further checked by means of X-ray topography techniques [86] at the European Synchrotron Radiation Facility (ESRF). Both techniques have a sensitivity of 1 dislocation over many $\mathrm{cm}^{2}$. The wafers with dislocation density lower than $1 / \mathrm{dm}^{2}$ were further processed.

\section{B. Crystal orientations and miscut angle}

Interaction of crystals with circulating beams exploits a few-micron-deep crystal portion, close to one of its surfaces [63]. Depending on the dynamics of a circulating beam, the angle between the optical surface of the crystal and its atomic planes $\left[\Theta_{m}\right.$ in Fig. 2(b)] might play a role in determining the channeling efficiency of the beam between the atomic planes of the crystal $[29,30,87,88]$. That angle is typically identified as the "miscut" or "off-axis" angle: it is mainly generated in the process of slicing the silicon ingots into silicon wafers, as a result of mechanical tolerances of the manufacturing equipment.

As shown in Fig. 2, any residual miscut implies that particles approaching the surface of the bent crystal with a small impact parameter may be only partially deflected, unable to reach the secondary absorber and possibly lost in the sensitive area of the accelerator.

The miscut angle must be kept as small as possible, and in any case should be much smaller than the angle subtended by the bent atomic planes. For example, collimation of the LHC circulating beam requires a crystal with a bending angle of $50 \mu \mathrm{rad}$ [63], translating in the need for a miscut angle in the order of few $\mu \mathrm{rad}$. This achievement requires a revisitation in the state of the art of manufacturing of silicon crystals. Indeed, the main fields which lead to the development of silicon crystals are the microelectronics (for industrial applications), and x-ray synchrotron facilities (scientific applications). For microelectronics, miscut angle of $\sim 1.7 \times 10^{4} \mu \mathrm{rad}$ is typically achieved and $\sim 1.7 \times 10^{3} \mu \mathrm{rad}$ is considered for demanding

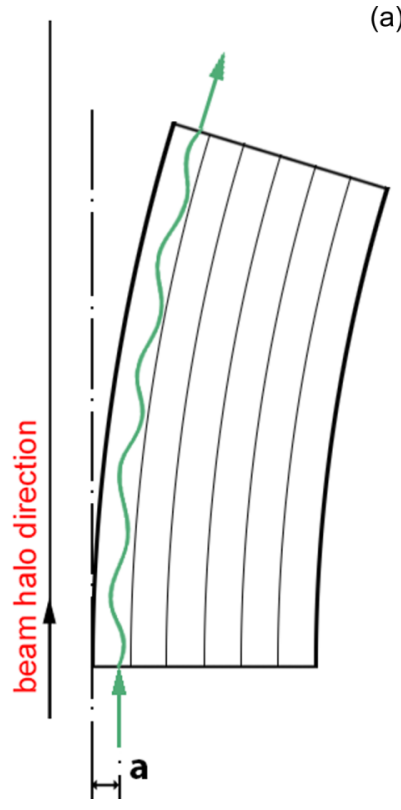

(a)

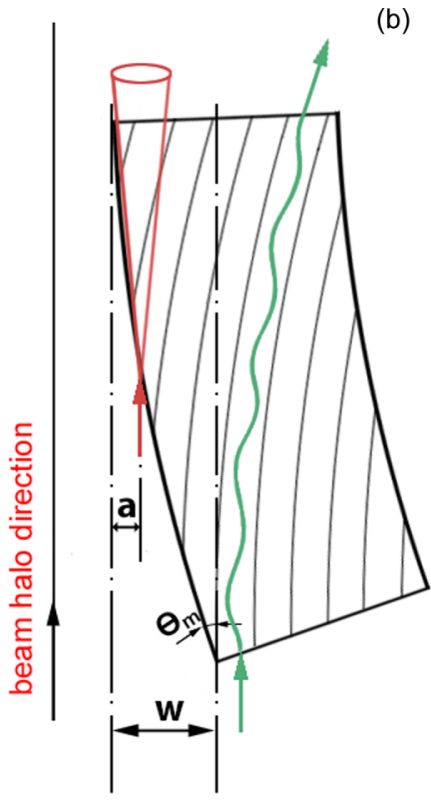

FIG. 2. (a) Sketch of a bent crystal with zero-miscut angle aligned to the halo of a beam circulating in a particle accelerator. Particles (green trajectory with an arrow) are captured in the channeling regime independently of their impact parameters to the crystal $a$. (b) A miscut angle $\Theta_{m}$ introduces a correlation between the impact parameter and the crystal-beam orientation. Only particles with sufficiently large impact parameter $w$ are captured under channeling regime and then steered. Particles with lower impact parameter (red arrow) interact with the crystal as if it was an amorphous target and their direction is spread out by multiple Coulomb scattering (red cone).

applications, while at $\mathrm{x}$-ray synchrotron facilities a miscut of $\sim 1.7 \times 10^{2} \mu \mathrm{rad}$ is considered as very noticeable.

Challenges in manufacturing of mm-size crystals with an ultrasmall miscut comes from the need of an ultra-accurate characterization setup and, mostly, from the availably of a polishing approach which accounts for unavoidable deformation of the crystal as a result of its fixing to the polishing equipment. To adjust the miscut, ultraprecise polishing techniques typically used for the manufacturing of ultraprecision optics were revisited to operate on silicon. The wafer's largest surfaces were indeed polished by means of magnetorheological finishing (MRF). MRF is a precision polishing method, mainly developed by QED Technologies (QED) $[89,90]$, to overcome many of the fundamental limitations of the traditional optics finishing. Conventional optical polishing uses stiff full-aperture laps to reduce the overall form error and delivers surfaces of low roughness (typically a few $\mathrm{nm}$ or less). While this process can work extremely well in delivering extremely flat or perfectly spherical surfaces, it fails as the surface shapes become more complex, e.g., aspheric or free-form, and hardly allows a precise control in the tilting of the sample to be polished. On the other hand, MRF is a deterministic finishing process, i.e., a process where the amount of material removal occurs in a highly predictable fashion, thus leading to high convergence rates and accurate estimates of the cycle times [91]. The MRF process is based on the use of a magnetorheological fluid, whose unique property is that 


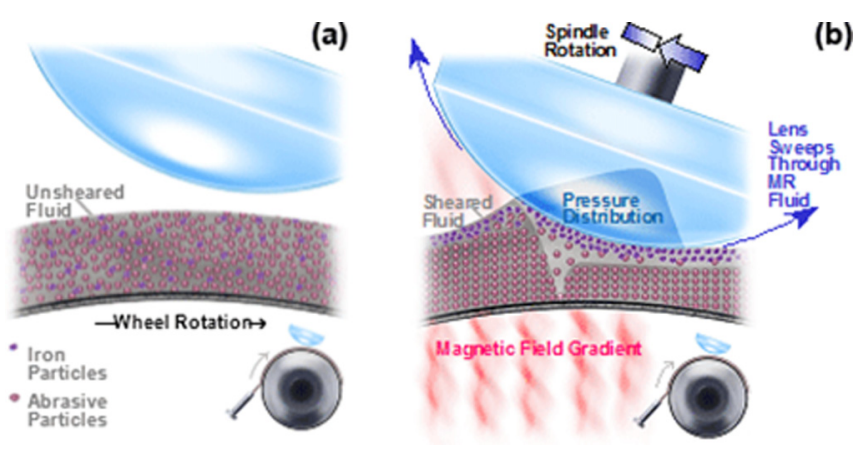

FIG. 3. (a) Creation of MRF polishing "spot." When the magnetic field is off, there is a random distribution of iron abrasive particles in the ribbon of fluid being transported by the rotating wheel. (b) When the magnetic field is turned on, the iron particles align and form chains giving to the fluid structure and stiffness. In addition, the water and the abrasives material move to the surface because the iron particles are attracted toward the wheel. When the workpiece is inserted into the fluid, the converging gap creates a highly sheared fluid layer that removes material with very low normal forces acting on the individual abrasive particles.

its viscosity changes by several orders of magnitude when it is introduced into a magnetic field, essentially turning from a liquid to a quasisolid in milliseconds (see Fig. 3).

The forces acting on the surface are predominantly tangential $[92,93]$. The normal forces on the individual abrasive particles are very small (limited to hydrostatic and kinetic). This contrasts with conventional polishing techniques where an abrasive material is forced into the surface through the action of a lap (either bound or loose). In that case normal forces can dominate, creating scratches, subsurface damage, and stress.

As a first step, the wafer surfaces were polished to reduce their flatness from a typical value of a few $\mu \mathrm{m}$ to less than 0.05 $\mu \mathrm{m}$ over their full area: surface topology was characterized with a Zygo 6" Verifire AT 1000 interferometer operating in Fizeau configuration (see Fig. 4). Subsequently, $\Theta_{m}$ was characterized by means of a high-resolution $\mathrm{x}$-ray diffractometer
(Panalytical X'Pert ${ }^{3}$ MRD XL) following the approach described in [94], and its value was decreased with successive polishing steps of MRF with miscut measurements. A record $\Theta_{m}$ value of less than $2 \mu \mathrm{rad}$ was achieved after a total of two iterations.

\section{Schemes for crystal bending}

The bending angle that the crystal must impose on the particle beam is determined by the needs and constrains of the experimental setup. In practical applications, it may vary from a few to some tens of $\mu \mathrm{rad}$, for example for the case of collimation [63] or extraction of the LHC [34,37] or FCC [55] circulating beam, to more than $10 \mathrm{mrad}$ for the case of experiments aiming to perform fixed-target experiments at the LHC [39-43,95,96].

Various approaches have been developed to achieve the needed deformation state: the most investigated are based on the action of a mechanical bender imparting the wanted deformation to the crystal. Alternative approaches are under investigation: they are based on the deposition of thin (few tens of nanometer thick) films generating stress in the crystal [97] or machining of a crystal surface to generate a superficially thin damaged layer under controlled conditions (ion implantation [98], sandblasting [99,100], grinding [101], surface grooving [102,103]). Independently from the method used to deform the crystal, it is important to avoid unwanted deformations of the crystal itself. In particular, bending angle of the crystal and torsional deformation (see Sec.III A ) must satisfy tolerances which are typically very tight. Moreover, in case of implementation of the crystal in a setup where the desired level of vacuum is achieved through thermal bake-out cycles (such as in the LHC), the deformational state of the crystal must be stable against such thermal cycles.

In the following we will focus on bending approaches based on the use of mechanical benders, as this approach currently is the most experimentally investigated.

For the case of experiments related to fixed target experiments in the multi- $\mathrm{TeV}$ regime, crystals capable of bending angle of various mrad are required [39-43,95,96]. This, jointly with the fact that at that scale energy channeling

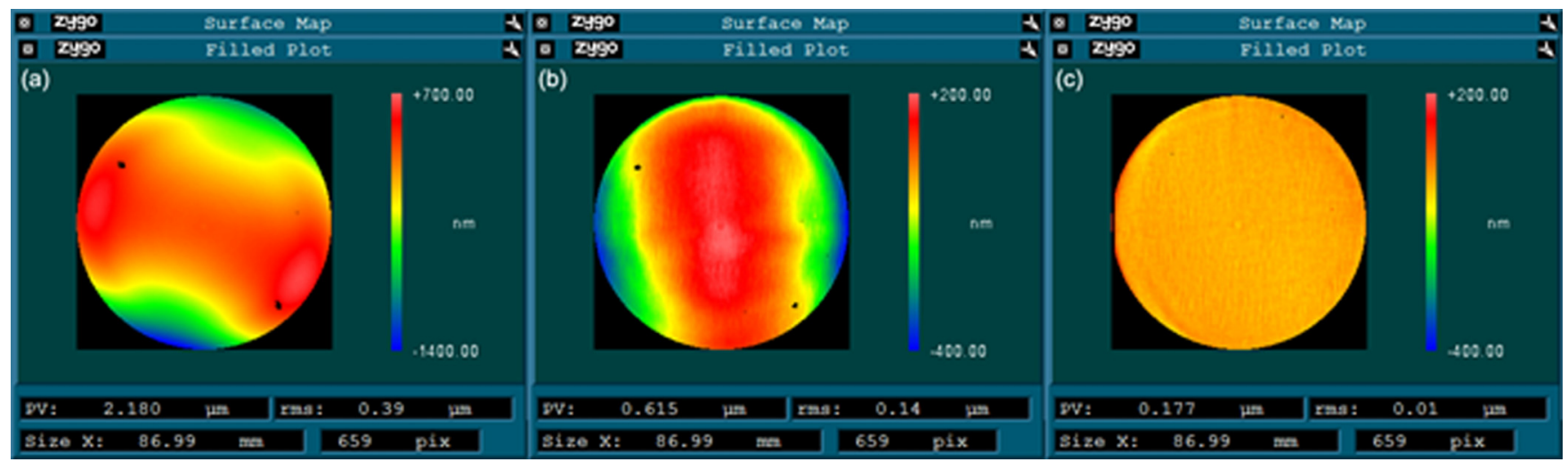

FIG. 4. (a) Interferometric characterization of a wafer surface prior to the polishing operations shows surface flatness of $0.39 \mu \mathrm{m}$. (b) After a first polishing run with a high removal rate MRF fluid, the surface flatness was improved to a value of $0.14 \mu \mathrm{m}$. (c) After a second polishing run using a low roughness fluid, surface flatness improved to $0.01 \mu \mathrm{m}$. Black dots in (a) and (b) are fiducials used to orient the wafer in the measuring step. 

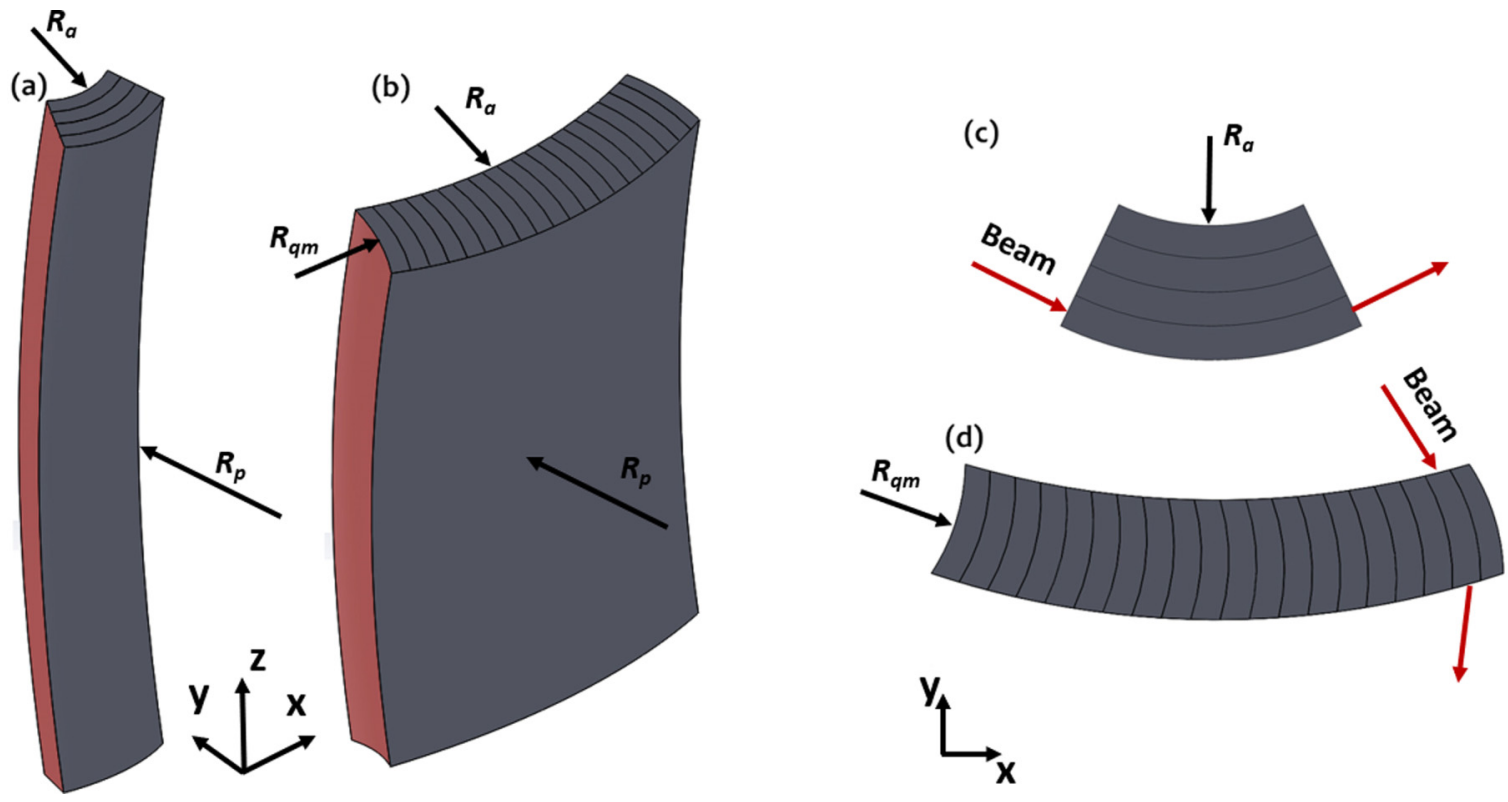

FIG. 5. (a) and (b) Silicon crystals bent under bending moments (not shown) acting at their ends. (b) and (c) Cross sections of the crystals taken at their middle along the $z$ axis. The crystal sketched in (a)-(c) exploits anticlastic deformation manifesting along the $x$ axis, occurring for any crystallographic orientation of the crystal. The crystal sketched in (b)-(d) exploits instead the so called "quasimosaic" deformation, occurring only under a proper choice of crystallographic orientations as a bending of the crystal planes along the smaller dimension of the crystal (in this case along the $y$ axis).

becomes inefficient at bending radii below few meters [5], results in crystals of a few $\mathrm{cm}$ thickness. Under such circumstances, crystals can be bent exploiting schemes which impart a "primary curvature" to the crystal. Typically, the crystal is clamped between the surfaces of a properly machined jaw (see Sec. IV A).

The case of applications expecting the interaction of a crystal with the halo of a circulating beam, such as beam collimation or extraction, requires crystals properly designed to reduce as much as possible beam losses. Compliance with this requirement typically results in crystals of thickness of some $\mathrm{mm}$ with bending radii of curvature larger than a few tens of meters. For such a case, exploitation of schemes based on a "primary curvature" is not feasible. Crystal bending relies on the use of secondary or tertiary deformations, arising because of a primary deformation imparted to the crystal through a mechanical bender. Two schemes are typically used: we consider a bar of an anisotropic material such as silicon, deformed under bending moments $(M)$ acting at both its ends (see Fig. 5). Displacement field along the $x, y$, and $z$ axis turns out to be [104]

$$
\begin{aligned}
u & =\frac{M}{2 I}\left(2 a_{13} x y+a_{36} y^{2}+a_{35} y z\right), \\
v & =\frac{M}{2 I}\left[-a_{13} x^{2}+a_{23} y^{2}+a_{33}\left(h z-z^{2}\right)-a_{35} x z\right], \\
w & =\frac{M}{2 I}\left[a_{35} x y+a_{34} y^{2}+a_{33} y(2 h-l)\right],
\end{aligned}
$$

where $a_{i j}$ are the components of the compliance matrix of the crystal, $I$ is the moment of inertia of the cross sections with respect the $x$ axis, and $h$ and $l$ are the crystal sizes along the $y$ and $z$ axis, respectively [see Fig. 5(a)].
The dependence of $v$ in Eq. (1) over $x^{2}$ shows that after the deformation, the crystal assumes a saddlelike shape, characterized by a "primary curvature" imparted by the mechanical bender, and a secondary curvature, owing to the "anticlastic deformation," characterized by an "anticlastic radius". The ratio between the anticlastic radius and the "principal radius" corresponds to the Poisson ratio of the crystal (i.e., $a_{33} / a_{13}$ ) in the $X Z$ plane. Being the Poisson ratio of any material between 0.5 and -1 , adjustment of principal radius of the crystal provides a fine adjustment of the anticlastic deformation. Moreover, a proper choice of crystallographic orientations leading to $a_{36} \neq 0$, thus resulting in a tertiary deformation, typically identified as "quasimosaic" [105] deformation (see Fig. 5), occurring as a bending of the planes along the crystal thickness.

The choice of bending mechanism influences the possible choices of the channeling planes. While anticlastic deformation manifests for crystals of any crystallographic orientation, quasimosaic deformation permits exploiting only (111) planes (or planes of higher Miller indices which would deliver low steering efficiency [106]). Moreover, quasimosaic deformation does not allow us to exploit axial channeling, which might be useful for future studies aimed at extraction or collimation in circular accelerators [107]. Due to such limitations, crystals exploiting anticlastic deformation are preferable.

\section{Crystals shaping}

Shaping of a crystal typically relies on a proper matching between chemical and mechanical methods, which we borrow from approaches well established in the field of silicon micromachining, and which we adapted to the manufacturing of macroscopic silicon crystals. 
The wafer previously processed was diced with a high precision dicing saw (Disco DAD 3220) to crystals whose lateral sizes are about $\sim 0.1 \mathrm{~mm}$ wider than the final desired size. Dicing parameters in terms of rotational speed, feed rate, blade grit, and thickness have to be optimized to reduce subsurface lattice damage induced in the crystal by the dicing operations as much as possible. Nevertheless a damaged layer extending for a few microns below the diced surface [27] is left after dicing. To recover the crystalline quality, purely chemical, chemical-mechanical polishing, or a mix between those techniques can be adopted to recover the crystalline quality of the surfaces which will be the entry/exit faces for the beam (chemical-mechanical polishing is employed as a last step for such situations where mirrorlike surfaces are needed). At the same time, crystal sizes are reduced from the $0.1 \mathrm{~mm}$ in excess.

For the case of crystals for collimation of the LHC beam, an optimal thickness of $4 \mathrm{~mm}$ and a bending angle of $50 \mu \mathrm{rad}$ were selected [63]. After being polished to decrease its miscut, a $2 \mathrm{~mm}$ thick wafer was diced to strips of $4.1 \times 55 \mathrm{~mm}^{2}$ lateral sizes. The lateral faces (sizes $2 \times 55 \mathrm{~mm}^{2}$ ) of the strips are parallel to the (110) face to within 1 deg. Subsequently to dicing, those faces were chemomechanical polished to remove the subsurface lattice damage and at the same time reduce crystal size along the beam from $\sim 4.1$ to $4.00 \pm 0.01 \mathrm{~mm}$.

\section{E. Characterization of crystalline quality}

A crucial aspect that crystals for applications in highenergy accelerators must satisfy is the absence of an amorphous or a crystalline-damaged layer on the crystal surface parallel to the direction of beam propagation. Indeed, the impact parameter of the beam with respect to the crystal is as small as a few $\mu \mathrm{m}$ [63]. Therefore, the crystalline quality of the surfaces hit by the beam is important to ensure a maximum channeling efficiency. Channeling must indeed occur starting from the very first atomic layers of a crystal $[108,109]$.

With the aim to characterize crystalline quality of the produced surfaces, an extensive experimental program was carried out using the most advanced methods typically used in semiconductors manufacturing.

Various authors already characterized subsurface damage and stress in amorphous materials treated by MRF $[92,110,111]$. However, a reliable and deep study of the crystalline quality of MRF-treated crystals through analysis techniques typically used to characterize crystalline materials is still missing. In particular, it should be pointed out that for a crystalline material there is no correlation between surface roughness and crystalline quality of the treated surface.

At first, high-resolution x-ray Bragg diffraction was used for a preliminary characterization of crystal surfaces in terms of dislocations or residual strain. As illustrated in Fig. 6, Bragg diffraction occurs when radiation, with a wavelength comparable to atomic spacing, is scattered in a specular fashion by the atoms of a crystal and undergoes constructive interference.

High-resolution diffraction of $\mathrm{x}$ rays is accomplished using a highly collimated and monochromatic beam and is routinely used worldwide to investigate deviations of a crystalline structure from an ideal crystal, which can be induced for

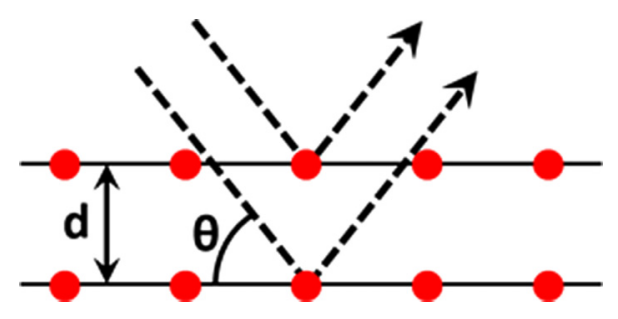

FIG. 6. Two x-ray beams with identical wavelength and phase approach a crystal and are scattered off two different atoms. The lower beam traverses an extra length of $2 d \sin \theta$. Constructive interference occurs when this length is equal to an integer multiple of the wavelength of the radiation. Such condition can be satisfied properly rotating the crystal with respect to the incident x-ray beam.

example by crystallographic defects, strain, or mosaicity. This technique foreseen orienting the crystal to the beam in such a way as to excite $\mathrm{x}$-ray diffraction and counting the number of diffracted $\mathrm{x}$ rays while rotating the crystal around such a position. Indeed, accounting for propagation and absorption of $\mathrm{x}$ rays by the crystal, it is expected that the diffraction occurs within a certain angular range. Recorded diffraction profiles (see Fig. 7) are typically called "rocking curves": a proper study of the profiles of such curves in terms of the dynamical theory of x-ray diffraction [112] reveals important information related to the perfection of crystal. For example, broadening of the rocking curve typically arises from a strained lattice, while a too high counting rate at the tails is a typical indication of the presence of dislocations.

A state-of-the-art X-ray diffractometer (Panalytical X'Pert ${ }^{3}$ MRD XL) operating with a $\mathrm{Cu}$ anode source installed at INFN-Ferrara was used to perform high resolution $\mathrm{x}$-ray diffraction characterization. In this machine, the $x$ rays generated from an x-ray tube are collected by a Göbel mirror [113], which delivers a beam with a divergence of about $0.02^{\circ}$. $\mathrm{X}$ rays coming from the mirror are injected into a compact monochromator exploiting four reflections on (220) oriented Ge crystals, and then conditioned to a size of $5 \times 0.2 \mathrm{~mm}^{2}$. The beam output from the monochromator has a typical divergence of $0.003^{\circ}$ and a wavelength relative spread less than $2 \times 10^{-5}$ and is used to probe the crystal surfaces in terms of the presence of dislocations, lattice strains, and relatively thick amorphous layers. Figure 7 shows the x-ray rocking curves of (220) plane, i.e., the second order diffraction from the (110) lattice plane, obtained on both the surfaces of the silicon crystal parallel to the particle beam and on the surface which represent the entry face for the beam, compared to the rocking curve recorded on a reference crystal. A comparison of these rocking curves demonstrates an extremely high quality for both the surfaces subjected to MRF and chemomechanical polishing. The presence of a number of dislocations in excess over the reference sample, possibly induced by the surface machining, would be revealed by the scattering tails around the main peak induced by the dislocation's deformation field. As can be noticed, no differences with respect to the tails of a reference crystal is detectable. Moreover, also the full-width half-maximum of the rocking curve $\left(\sim 0.003^{\circ}\right)$ coincided with the values expected for a case free from defects such as strains or mosaicity. 

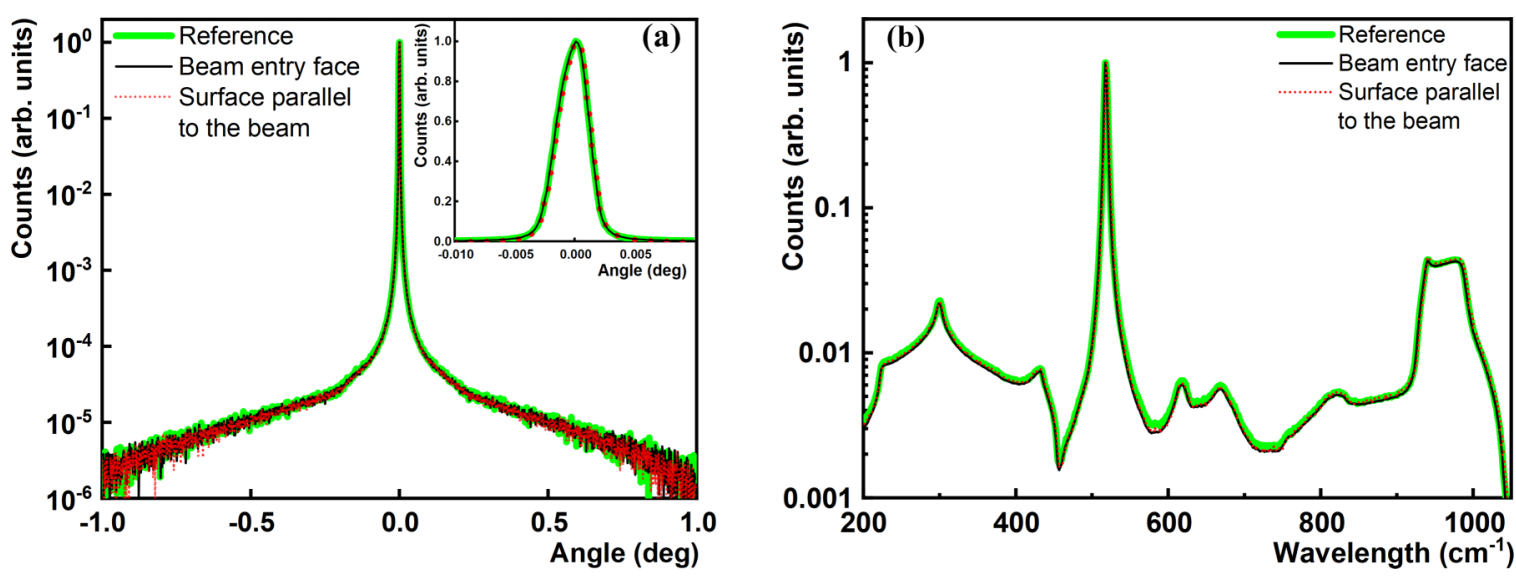

FIG. 7. (a) High resolution x-ray diffraction rocking curves recorded on the surface of the crystal parallel to the propagation of the beam (red dashed curve), on the beam entry face (black curve), and on a reference surface (green curve). Absence of deviation of the tails from an ideal profile is indication of the absence of dislocations. The inset shows a zoom of the rocking curves in the central region. Full-width half-maximum of the rocking curves are identical to the ones of a reference, indicating the absence of strained layers (b) micro-Raman spectra collected on the crystal surface parallel to the beam (red dotted curve) and on the beam entry face (black line) are compared to micro-Raman spectra recorded on a reference sample (green). The crystal surface results to be free from strain or amorphous components, which would appear as additional peaks.

Operating with an $8 \mathrm{keV} x$-ray beam, we obtained a signal which is averaged over $\sim 13 \mu \mathrm{m}$ below the crystal surface. To obtain information from a thinner region below the surface, we used micro-Raman spectroscopy [114,115]. This technique relies upon inelastic scattering of photons and is typically used to determine vibrational modes of molecules or atoms in a crystal. The properties of the vibrational modes of molecules or atoms in a lattice are largely determined by the mass of the atoms and their bond type. The appearance of any physical factor affecting the short-range order results in modifications in the vibrational characteristics of the atoms and are readily noticeable in the Raman spectrum. This reflects, for instance, in striking differences between the spectrum of an amorphous material and of a crystalline sample of the same kind.

In the setup we used, a laser of $532 \mathrm{~nm}$ wavelength was focused to a spot of a few $\mu \mathrm{m}$. The chosen wavelength probes a thickness of $\sim 1.3 \mu \mathrm{m}$ [116] under the crystal surfaces. The laser light interacts with atomic vibrations, phonons, or other excitations in the system, resulting in the energy of the laser photons being shifted. The shift in energy gives information about the vibrational modes in the system. Results of the characterizations, reported in Fig. 7(b), highlight the presence of only a crystalline phase of silicon, manifesting with peaks at $\sim 303$ and $\sim 520 \mathrm{~cm}^{-1}$ and the absence of other crystalline phases or strained structures, which would manifest as additional peaks in the recorded spectrum [114,115].

To further investigate the crystalline quality, we also used Rutherford backscattering in channeling condition (c-RBS) to get information related to the atomic ordering of the first atomic layers of both faces of the crystal which are parallel to the beam and to the beam entry face. c-RBS was carried out using $2.0 \mathrm{MeV}{ }^{4} \mathrm{He}^{+}$at a scattering angle of $160^{\circ}$ in IBM geometry at INFN-Legnaro laboratories. Surface $\chi_{\min }$, defined as the ratio of the RBS yield under channeling alignment and the yield in random orientation extrapolated at the surface channel, was chosen as a quantitative parameter. The higher the degree of crystalline order in the lattice, the lower the value of $\chi_{\min }$ on the surface due to the reduction of dechanneling from the defects in the crystal. c-RBS provides information on the presence of crystalline defects up to a depth of $\sim 2 \mu \mathrm{m}$ with a few nanometer resolution in the crystal depth. Both surfaces recorded a value for $\chi_{\min }$ that is compatible with the value recorded on a crystal with a surface free from crystallographic defects. Moreover, simulations allowed us to quantify the surface peak areal density of $\mathrm{Si}$ atoms that do not contribute to channeling. It is worth to notice that even for perfect crystals the $\mathrm{Si}$ atomic areal density corresponding to this peak is $1 \times 10^{16}$ atoms $/ \mathrm{cm}^{2}$ $[117,118]$. This is because at this energy the beam focusing due to channeling needs to cross a certain crystal thickness before starting to decrease the backscattering yield. Mapping the crystal surface on both beam entrance and lateral faces with ${ }^{4} \mathrm{He}^{+}$spots $1 \times 1 \mathrm{~mm}^{2}$ gave surface peak values from $1 \times 10^{16}$ to $1.4 \times 10^{16}$ atoms $/ \mathrm{cm}^{2}$. This means that a noncrystalline surface $\mathrm{Si}$ fraction can be present as low as from 0 to $0.8 \mathrm{~nm}$ over the sample surfaces, reasonably in the form of a $\mathrm{SiO}_{2}$ nanolayer that naturally forms after air exposure $[117,118]$

The high order of crystalline perfection was confirmed also by high resolution transmission electron microscopy (HRTEM). That is a technique where a beam of electrons is transmitted through a specimen to form an image of the crystallographic structure of a sample at an atomic level. Also in this case, characterizations highlight an ordered arrangement of atomic columns preserved up to the crystal surfaces [see Figs. 8(c) and 8(d)].

\section{CRYSTAL DEFORMATION AND CHARACTERIZATION}

\section{A. Control of crystal elastic deformation}

The largest scientific field employing bent crystals is $\mathrm{X}$-ray diffraction. The state-of-the-art in controlled 

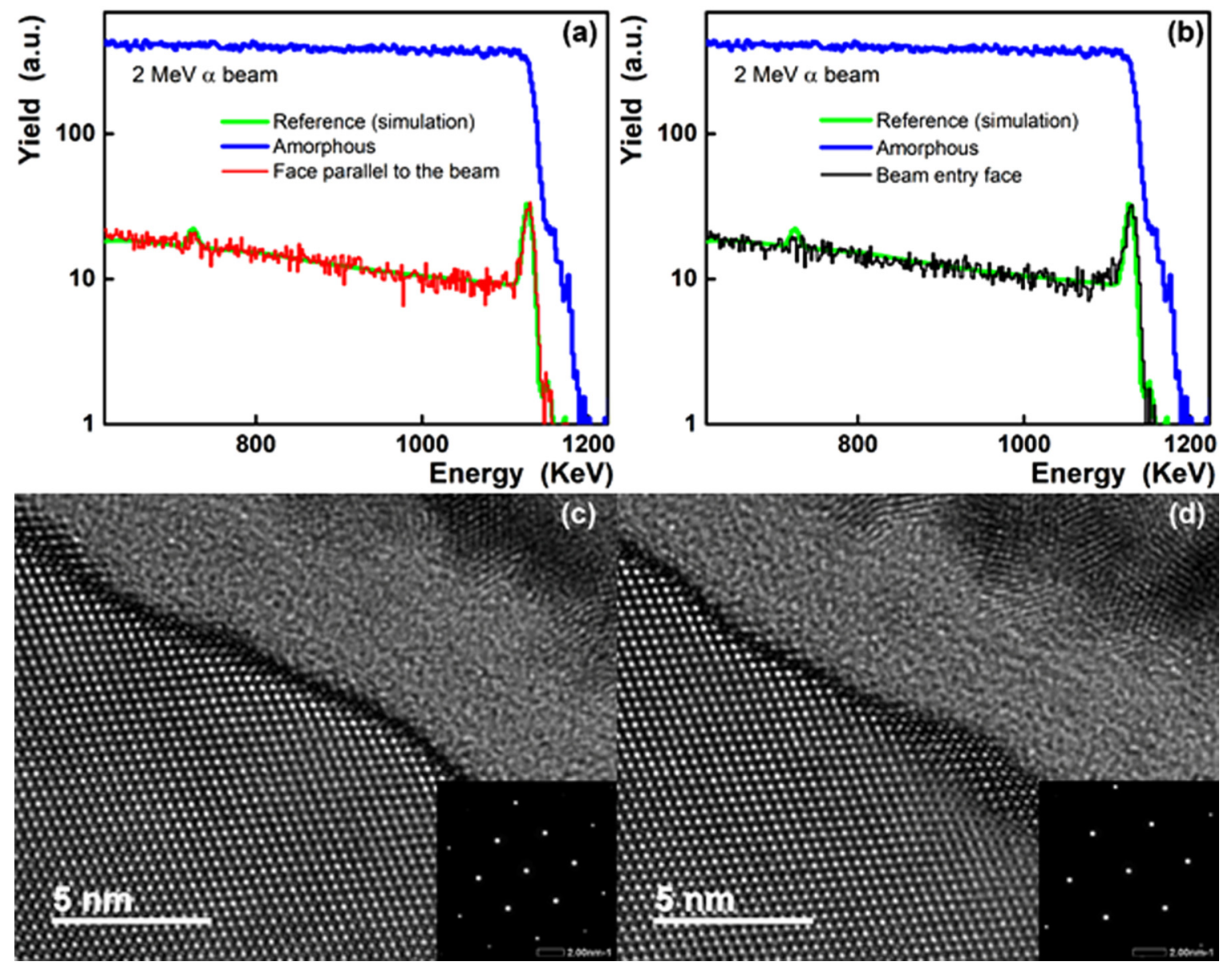

FIG. 8. (a) and (b) c-RBS spectra of $2 \mathrm{MeV}^{4} \mathrm{He}^{+}$recorded respectively on the crystal face parallel and perpendicular to the direction of propagation of a beam in a circular accelerator. (c) and (d) TEM characterizations for the same surfaces. The insets show the electron diffraction pattern of the sample. Both surfaces were found to be free from subsurface lattice damage induced by MRF and chemical-mechanical polishing operations, respectively.

deformation of silicon crystals is nowadays dictated by technologies employed worldwide at synchrotrons, where controlled deformation of crystals, typically $\mathrm{Si}$ or Ge crystals, is routinely accomplished through mechanical benders actuated by motors. This choice allows compensating mechanical imperfections which arise in mechanical manufacturing of the benders or of the crystals and delivers crystals with a deformational field typically controlled within a few $\mathrm{nm}$ at the region of interaction between the crystal and the x-ray beam. On the other hand, for the case of the most modern particle accelerators, the usage of motor-actuated benders might represent a relevant risk. Failure of a motor might indeed compromise a key component of a setup, and its replacement would be an operation far from trivial, as the crystal and its bender would operate in an area not easily accessible, given the high radioactive environment. The material of the bender must be compatible for operations in an ultrahigh vacuum environment and should not lead to electron cloud activity in the accelerator $[119,120]$. Moreover, the assembly must be as light as possible: at ultrahigh energies, such as for the case of the LHC or future accelerators, the critical angle for channeling scales down to values as low as a few $\mu$ rad or less and as a consequence the crystal must be aligned to the beam with sub- $\mu$ rad accuracy. The most reliable technology that can achieve this accuracy is based on the use of piezoactuated goniometers [121], whose performances (as for any piezo-actuated motor) are maximized as they operate under the lowest possible weight. This is an additional reason to avoid the use of a motor-actuated bender, as it would likely result in a bulky device. Adoption of a static bender capable of imparting the correct deformational state to the crystal is often the most promising approach, even if mechanical tolerances are often extremely challenging.

A material satisfying the briefly listed requirements is titanium "grade 5," an alloy composed of 90\% titanium, 6\% aluminum, and $4 \%$ vanadium. This material, thanks to its low density and extremely high strength, typically finds applications for aerospace, naval, and biomechanical applications, engine components, and sport equipment, but is very rarely used in ultrahigh precision mechanics due to its poor machinability [122].

For the case of crystals with a thickness of a few $\mathrm{cm}$ and radius of a few meters, the possibility to bend them through clamping of the crystal between properly shaped surfaces of a jaw (see Fig. 9) is under study. Crystals must operate at an energy range in the $\mathrm{TeV}$ region and this requires an extremely high uniformity of the bending radius along the crystal: control of deformational state of the crystal relies 


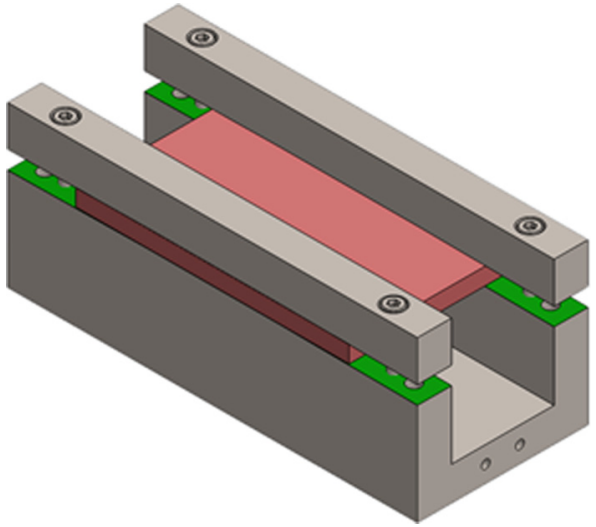

FIG. 9. Representation of a bent crystal with a geometry suitable for fixed-target experiments in the LHC. The crystal (red part) is clamped between surfaces of a bender with surfaces (green) machined to a cylindrical shape.

on perfection of machining of the surfaces of the bender in contact with the crystal and the surfaces of the crystal itself. That is an approach already developed for extraction of the beam circulating in the SPS [123], which needs to be refined to improve the uniformity of crystal deformations.

For the case of crystals under preparation for studies of the collimation of the LHC circulating beam, we see crystals exploiting anticlastic deformation as the most promising. This approach were initially developed at the Institute for High Energy Physics (Protvino, Russia) [26], and further refined at the INFN-Ferrara (Ferrara, Italy).

Benders are based on revisiting technologies employed for the preparation of bent crystals for the steering of highenergy protons or ions circulating in the SPS [29-31,88,124], its extracted lines [125-127], and Tevatron [3], and initially developed at the Institute for High Energy Physics (Protvino, Russia) [26]. The previous generation of bender manufactured at INFN-Ferrara were made of aluminum alloys [128] for easy machinability. Now, benders are manufactured in titanium alloys and mechanisms allowing for adjustment of the crystal deformational state in terms of torsion [129] and bending radius are removed, resulting in a geometrically simpler and a lighter support.

Reaching the correct deformational state of the crystal purely relies on ultrahigh precision machining processes of the surfaces in contact with the crystal: with the aim to exploit "anticlastic deformation," a "c-shaped" bender was manufactured (see Fig. 10), where surfaces in contact with the crystal must have a very well controlled inclination. The requirement on bending angle of $50 \pm 2.5 \mu \mathrm{rad}$ translates in a tolerance for the inclination around the $y$ axis of only $0.0022^{\circ}$.

Aside from imparting a proper bending to the crystal, the bender must avoid generation of unwanted deformations, such as torsional effects. To illustrate the role of torsion for simplicity, we refer to the scheme, already described, exploiting anticlastic deformation (see Fig. 11); similar considerations apply to other possible geometries. The presence of crystal torsion introduces a rate of change of the optimal angle of crystal-beam alignment for unit displacement along the vertical direction of the crystal (see Fig. 11). This reduces
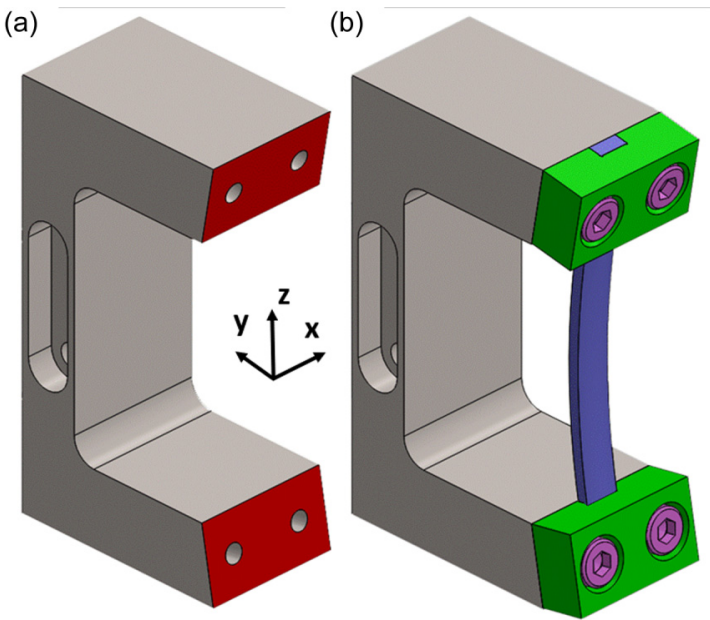

FIG. 10. (a) Base structure of a static bender for strip crystals. Red colored surfaces are tilted by $89.9529 \pm 0.0022^{\circ}$ around the $y$ axis toward the inner side of the bender to impart a "primary bending." The same surfaces are tilted of less than $0.0020^{\circ}$ around the $z$ axis to avoid torsion (in this picture the tilting around the $y$ axis is largely increased). (b) Crystal (blue color) assembled on the bender device (deformation of the crystal is exaggerated on purpose in the picture). A couple of clips (green color) clamp the crystal on the holder. Screws (violet color) secure the clamps to the bender.

the geometrical acceptance of the crystal and its steering efficiency, resulting in a degradation of its performances as it operates in a circular accelerator [30,31].

For the case of crystal/benders assemblies for collimation of the LHC, crystal torsion might arise as a consequence of mechanical imperfections of the holder or inaccuracies in the procedure of assembly of the crystal on the holder. Referring to Fig. 10, the bender must be machined with approaches delivering small mutual rotations around the $z$ axis of the surfaces in contact with the crystal: thanks to recent developments, a tolerance of $0.0020^{\circ}$ (leading to a torsion of $1 \mu \mathrm{rad} / \mathrm{mm}$ ) is nowadays reachable and if needed, lower values for torsion can be reached through further developments in holder machining or using benders exploiting hinged flexure mechanisms specifically designed to reduce torsion $[26,129]$

\section{B. Characterization of crystal deformational state}

Prior to installation in an accelerator, the crystal must be properly characterized in terms of its deformational state: tolerances in terms of bending and torsion are indeed challenging to achieve, and inaccuracies in the machining of benders or in the mounting procedure may result in an unwanted deformational state of the crystal.

A preliminary characterization of the deformational state of the crystal was performed using a white-light interferometer (Veeco NT1100). This characterization delivered precise information related to the surface of the crystal. In principle, such characterization could be related to the deformational state of the atomic planes, nevertheless we prefer to perform directed characterizations of the deformational state of atomic planes using a high-resolution $\mathrm{x}$-ray diffractometer. 


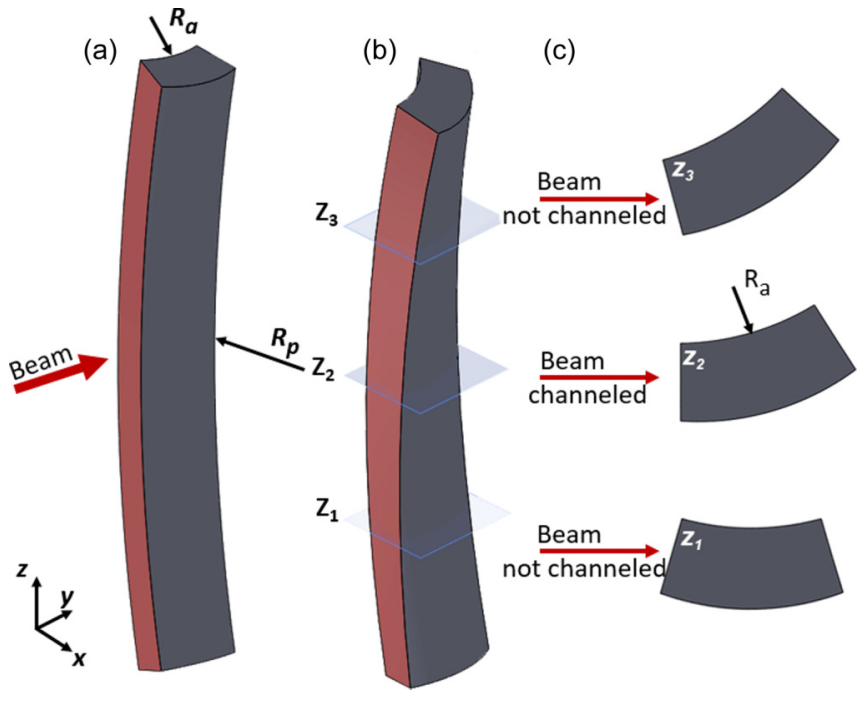

FIG. 11. (a) A bent silicon strip crystal. A couple of moment are applied at end of the crystal, resulting in a "primary" bending of the crystal, with radius $R_{p}$, and a "anticlastic deformation" of crystal cross section, manifesting with a curvature of radius $R_{a}$. (b) As a consequence of mechanical imperfections of the holder or of the mounting procedures, the bent crystal might be subject to a torsion. (c) Cross sections of a bent strip subjected to torsion, taken at three different $z$ positions. As a result of the torsion, alignment between crystal cross section and the beam linearly changes along the vertical direction of the crystal, reducing its geometrical acceptance.

The instrument was interfaced to a custom-made autocollimator to increase its accuracy and precision in a wide class of measurements, among which the characterization of the crystal deformational state. Measurements of bending angle were achieved by mounting the crystal on the Eulerian cradle of the diffractometer: six degrees of freedom allow the alignment of the crystal to the x-ray beam. Figure 12 sketches the method for the measurement of crystal bending angle. The crystal is oriented to the beam in order to obey the Bragg reflection condition from the bent lattice planes at its central region $\left(X_{2}\right)$. Deformations of the crystalline planes break the translational symmetry of the system along the $X$ axis, establishing a linear relationship between the position along the $X$ axis and the angle at which the crystal must be oriented to the beam in order to match the Bragg reflection.

Characterization of the deformational state of the crystal is accomplished by translating the crystal with respect to the beam along the $X$ axis and recording an x-ray rocking curve at each position. The center of mass of each rocking curve is calculated and a map relating centroid of each rocking curve to the $X$ position is generated [inset of Fig. 12(b)], delivering exact information on the deformational state of the crystal.

The measuring setup is strongly influenced by precision and accuracy of the angular motions. With the aim to precisely characterize angular motion of the crystal during rotations around the $\Omega$ axis and to characterize parasitic rotations around the same axis occurring due to mechanical tolerance when the crystal is translated, a laser autocollimator tracks angular motion of a mirror integrated with the crystal. Ultimately, accuracy and precision reached by the autocollimator determines angular accuracy and precision in the reconstruction of the deformational state of the crystal. A first-generation setup employed an autocollimator capable of $1 \mu \mathrm{rad}$ precision (a)

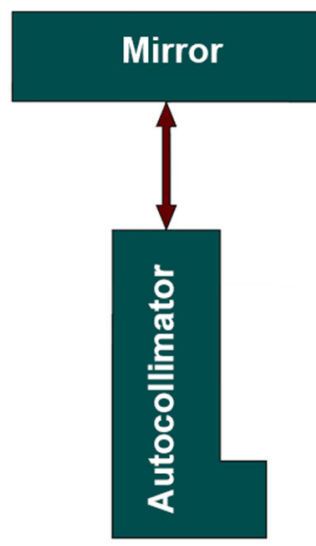

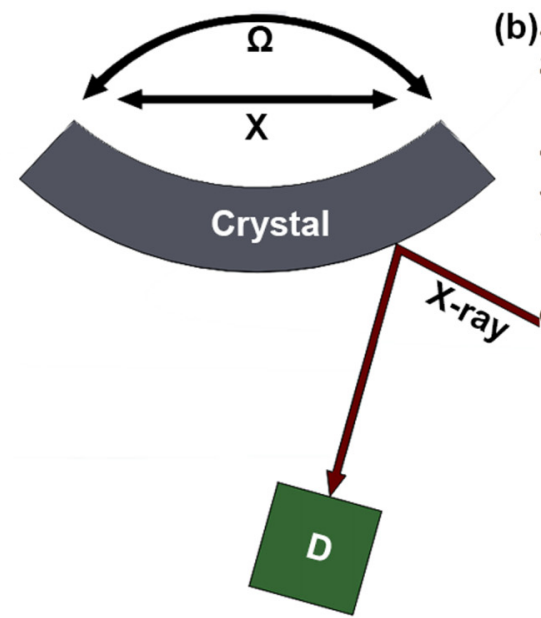

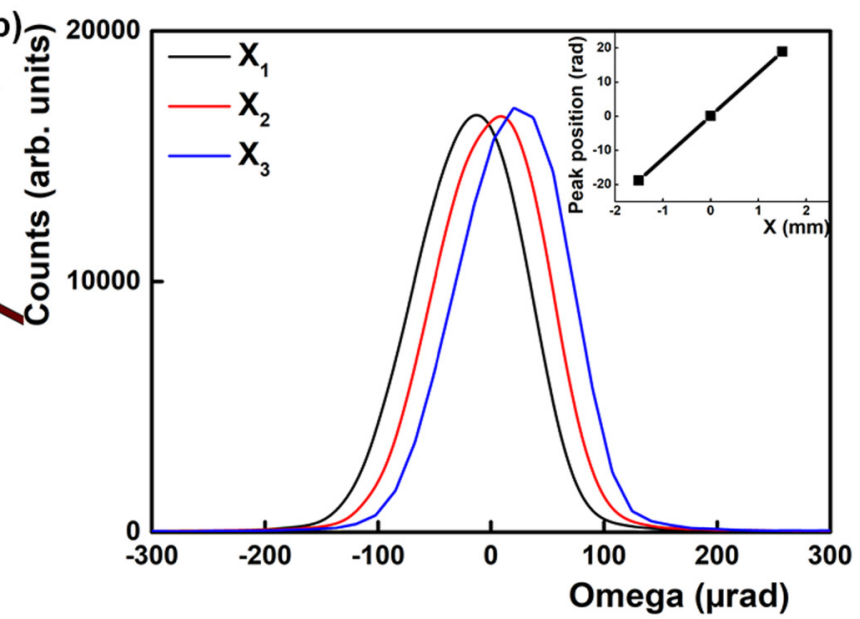

FIG. 12. (a) Sketch of the setup employed to characterize the deformational state of the crystal in terms of the bending of the lattice planes. A small portion of the crystal is illuminated with a collimated and monochromatic x-ray beam. The crystal is oriented with respect to the beam in order to match the Bragg reflection condition from its bent planes. The high order reflection (440) is chosen to minimize footprint of the x-ray beam on the crystal: Bragg reflection occurs as the angle between lattice planes and x-ray beam is $53.3508^{\circ}$. The rotation of the crystal around the $\Omega$ axis produces a "rocking curve" [see profiles in (b)]. The bending of the crystal breaks the translational symmetry of the system along the $\mathrm{x}$ axis: if the crystal is aligned to the beam in order to match Bragg reflection, alignment is lost after translation of the crystal. Angular motions occurring while rotating the crystal or as result of mechanical tolerance occurring during the translation along the $X$ axis are characterized by means of an autocollimator tracking a mirror integrated to the crystal. (b) Recording a set of rocking curves at various positions while translating the crystal along the $X$ axis delivers a map of the crystal deformational state. The center of mass of each rocking curve is calculated and plotted against the corresponding position along the $X$ axis: the slope of the linear relationship between the $X$ position and the center of mass of the corresponding rocking curves gives a measurement of the bending angle of the crystal. 
TABLE I. Comparison between bending angles measured through high-resolution $\mathrm{x}$-ray diffraction and those measured in channeling experiments for a large set of crystals. In all the cases the bending angle measurements with the cited techniques are in a good agreement.

\begin{tabular}{lrc}
\hline \hline $\begin{array}{l}\text { Crystal } \\
\text { code }\end{array}$ & $\begin{array}{c}\text { Bending angle } \\
\mathrm{x} \text { ray }(\mu \mathrm{rad})\end{array}$ & $\begin{array}{c}\text { Bending angle } \\
\text { particle channeling }(\mu \mathrm{rad})\end{array}$ \\
\hline STF47 & $33 \pm 2$ & $34[130]$ \\
STF48 & $144 \pm 2$ & $144[130]$ \\
STF49 & $247 \pm 3$ & $247[130]$ \\
STF50 & $142 \pm 5$ & $139[130]$ \\
STF51 & $33 \pm 2$ & $33[130]$ \\
STF70 & $56 \pm 2$ & $54[131]$ \\
STF71 & $60 \pm 5$ & $61[131]$ \\
STF99 & $119 \pm 3$ & $120 \pm 2[80]$ \\
STF100 & $67 \pm 6$ & $63 \pm 2[80]$ \\
STF101 & $170 \pm 6$ & $165 \pm 2[80]$ \\
STF102 & $45 \pm 3$ & $42 \pm 2[132]$ \\
STF103 & $52 \pm 5$ & $55 \pm 2[80]$ \\
STF105 & $49 \pm 3$ & $50 \pm 2[80]$ \\
STF106 & $42 \pm 2$ & $40 \pm 2[133]$ \\
STF107 & $55 \pm 2$ & $55 \pm 2[133]$ \\
STF114 & $52 \pm 3$ & $52 \pm 2[134]$ \\
\hline \hline
\end{tabular}

and accuracy; more recently this tool has been upgraded to be capable of a $0.5 \mu \mathrm{rad}$ accuracy and precision.

The measurement setup has been benchmarked in terms of crystal bending angle against a wide series of experiments involving the use of bent crystals as elements in the steering of high energy particle beams. Those experiments were performed at H8 and H4 external line of the SPS of CERN. Table I reports a comparison of the values of the bending angles determined at our laboratory with those determined at the experimental setups exploiting particle channeling. The comparison highlights the reliability of the characterizations conducted with a laboratory equipment.

\section{CRYSTALS THERMAL STABILITY}

For the cases where the crystal must operate under ultrahigh vacuum, a key parameter that the bender-crystal assembly must satisfy is the stability of its deformational state with respect to bake-out cycles which are needed to reach the working condition. For the case of LHC, the achievement of the operational conditions was accomplished through a series of bake-out cycles which brings the crystal and the bender at $250^{\circ} \mathrm{C}$ for $48 \mathrm{~h}$ for each cycle [135].

In order to assure thermal stability of the bender material, a series of thermal annealing were performed on the titanium material prior to and after its machining process. We assume thermal stability of the assemblies if the crystal does not change its deformational state after at least ten vacuum bakeout cycles: the crystal deformational state was characterized before and after each thermal cycle.

Figure 13 reports the measured bending angle and torsional values for a crystal subject to a total of 50 thermal cycles, highlighting the robustness of the assembly against these thermal cycles.

\section{CONCLUSIONS}

Thanks to their well-ordered atomic structure, crystalline materials have been studied for a few decades as elements to steer particle beams at particle accelerators. As a result of considerable technological advances, their applications at ultrahigh energy and intensity accelerators is now possible. Such a result is achieved thanks to a highly interdisciplinary approach developed at INFN-Ferrara merging the most advanced techniques typically used in the fields of silicon micromachining, ultraprecise optics manufacturing, $x$-ray topography, and ultraprecise metrology.
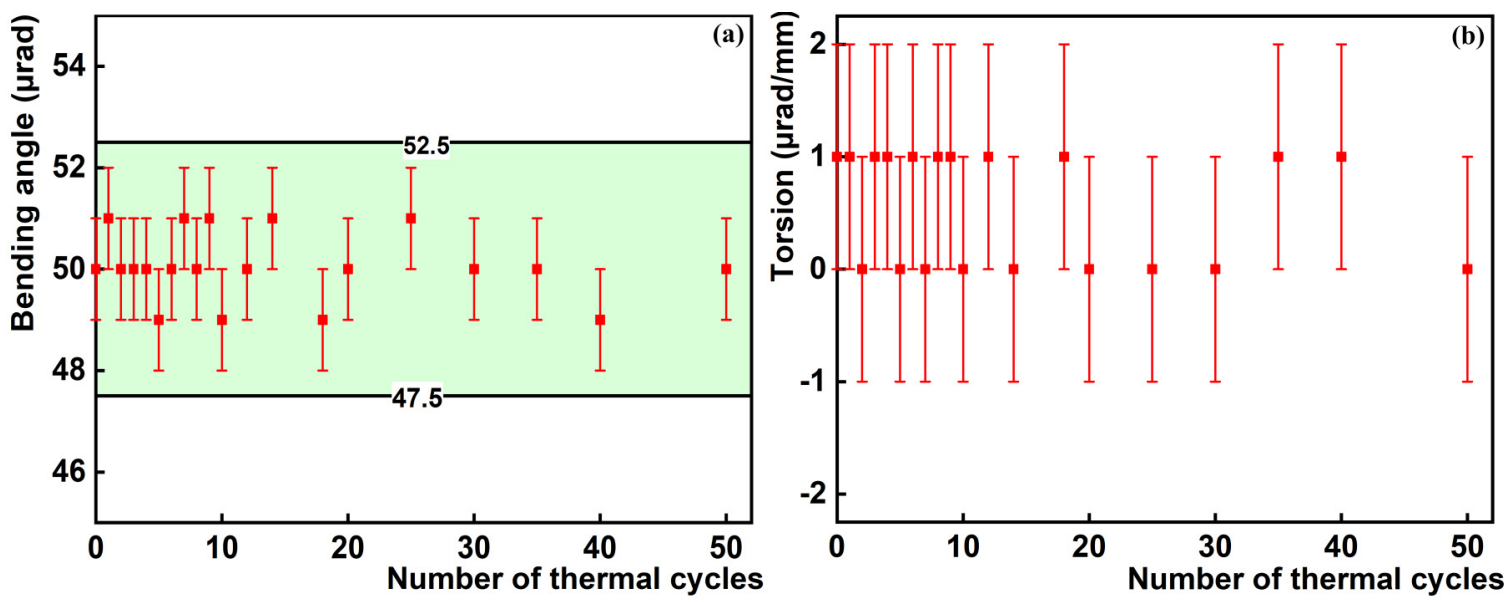

FIG. 13. Study of the thermal stability of the crystal against bake-out thermal cycles. (a) Value of bending angle vs the number of thermal cycles. Green area highlights the region of acceptable bending angle of the crystal following [63]. (b) Measured torsion as a function of the number of thermal cycles. The stability of the bending angle and torsion values demonstrates the robustness of the crystal deformation state and of the bender. 
The results of laboratory-based investigations highlight the crystallographic perfection of the crystals starting from their first few atomic layers, an ultralow miscut angle of just few $\mu \mathrm{rad}$ and a reliable correlation between the deformational state of the crystal characterized by means of high-resolution $\mathrm{x}$-ray diffraction and the channeling of high energy particle beams.

After a long lasting experimental investigation carried at CERN mainly in the frame of the UA9 collaboration, this technology has been integrated into the HL-LHC baseline program for the realization of a crystals-based setup for collimation for heavy-ion beams. At the same time, availability of this technology opens new possibilities also for beam extraction and to perform innovative fixed-target experiments at the most advanced particle accelerators.

Besides that, crystals might play a key role also to achieve slow extraction of particle beams circulating in lower energy accelerators such as the SPS at CERN or at synchrotrons facilities, as innovative sources of gamma radiation, and as elements useful for the production of positron or beams to be used in a wide class of physics investigations.

\section{ACKNOWLEDGMENTS}

We acknowledge Professor Lucio Rossi and Dr. Walter Scandale for their long-lasting support to this activity in the frame of the UA9 collaboration. A. Mazzolari acknowledge Professor Lucio Rossi, Dr. Stefano Redaelli, Dr. Simone Gilardoni, Dr. Alessandro Masi, Dr. Alexander Taratin, Professor Victor Tikhomirov, Dr. Valery Biryukov, and Professor Geoffrey Hall for many stimulating conversations on the subjects treated in the manuscript and for critically reading it. A. Mazzolari acknowledges Andrea Persiani and Claudio Manfredi (PERMAN), Dr. Claudio Sgarbanti (Biomeccanica), and Dr. Riccardo Signorato (CINEL) for their friendly support with manufacturing of crystal benders. This work has been supported by the ERC Consolidator Grant CRYSBEAM GA, the ERC Consolidator Grant SELDOM GA 771642, and by the INFN CSN1. A. Mazzolari and V. Guidi acknowledge founding from PRIN 2015LYYXA8 "Multi-scale mechanical models for the design and optimization of micro-structured smart materials and metamaterials." The authors would like to thank to BM05 staff and ESRF facility for the beamtime access for x-ray diffraction imaging experiments at BM05 beamline.
[1] A. Baurichter, K. Kirsebom, R. Medenwaldt, S. P. Moller, T. Worm, E. Uggerhoj, C. Bino, M. Clement, N. Doble, K. Elsener, L. Gatignon, P. Grafstrom, U. Mikkelsen, A. Freund, Z. Vilakazi, P. Siffert, and M. HageAli, New results from the CERN-SPS beam deflection experiments with bent crystals, Nucl. Instrum. Methods Phys. Res. Sect. B 119, 172 (1996).

[2] C. T. Murphy, R. Carrigan, D. Chen, G. Jackson, N. Mokhov, H. J. Shih, B. Cox, V. Golovatyuk, A. McManus, A. Bogacz, D. Cline, S. Ramachandran, J. Rhoades, J. Rosenzweig, B. Newberger, J. A. Ellison, S. Baker, C. R. Sun, W. Gabella, E. Tsyganov, A. Taratin, A. Asseev, V. Biryukov, A. Khanzadeev, T. Prokofieva, V. Samsonov, and G. Solodov, First results from bent crystal extraction at the Fermilab Tevatron, Nucl. Instrum. Methods Phys. Res. Sect. B 119, 231 (1996).

[3] D. Still et al., Recent T980 crystal collimation studies at the tevatron exploiting a pixel detector system and a multi-strip crystal array in Proceedings of IPAC2012 (2012).

[4] R. A. Carrigan, D. Chen, G. Jackson, N. Mokhov, C. T. Murphy, S. Baker, A. Bogacz, D. Cline, S. Ramachandran, J. Rhoades, J. Rosenzweig, A. Asseev, V. Biryukov, A. Taratin, J. A. Ellison, A. Khanzadeev, T. Prokofieva, V. Samsonov, G. Solodov, B. Newberger, E. Tsyganov, H. J. Shih, W. Gabella, B. Cox, V. Golovatyuk, and A. McManus, Beam extraction studies at $900 \mathrm{GeV}$ using a channeling crystal, Phys. Rev. Special Topics 5, 043501 (2002).

[5] V. M. Biryukov, Y. A. Chesnokov, V. I. Kotov, and V. M. Biryukov, Crystal Channeling and Its Application at HighEnergy Accelerators (Springer, Berlin 2010).

[6] W. Scandale, G. Arduini, M. Butcher, F. Cerutti, M. Garattini, S. Gilardoni, A. Lechner, R. Losito, A. Masi, D. Mirarchi, S. Montesano, S. Redaelli, R. Rossi, P. Schoofs, G. Smirnov, G. Valentino, D. Breton, L. Burmistrov, V. Chaumat, S. Dubos, J. Maalmi, V. Puill, A. Stocchi, E. Bagli, L. Bandiera,
G. Germogli, V. Guidi, A. Mazzolari, S. Dabagov, F. Murtas, F. Addesa, G. Cavoto, F. Iacoangeli, L. Ludovici, R. Santacesaria, P. Valente, F. Galluccio, A. G. Afonin, Y. A. Chesnokov, A. A. Durum, V. A. Maisheev, Y. E. Sandomirskiy, A. A. Yanovich, A. D. Kovalenko, A. M. Taratin, A. S. Denisov, Y. A. Gavrikovi, Y. M. Ivanov, L. P. Lapina, L. G. Malyarenko, V. V. Skorobogatov, T. James, G. Hall, M. Pesaresi, and M. Raymond, Observation of channeling for $6500 \mathrm{GeV} / \mathrm{c}$ protons in the crystal assisted collimation setup for lhc, Phys. Lett. B 758, 129 (2016).

[7] E. N. Tsyganov, Fermilab TM-682 (1976).

[8] V. Fanti, A. Lai, D. Marras, L. Musa, A. Nappi, R. Batley, A. Bevan, R. S. Dosanjh, R. Galik, T. Gershon, B. Hay, G. E. Kalmus, S. Katvars, C. Lazzeroni, R. Moore, D. J. Munday, M. D. Needham, E. Olaiya, M. A. Parker, M. Patel, M. Slater, S. Takach, T. White, S. A. Wotton, E. Bal, G. Barr, G. Bocquet, J. Bremer, R. Brodier-Yourstone, P. Buchholz, M. Burns, A. Ceccucci, M. Clement, T. Cuhadar-Donzelsmann, D. Cundy, N. Doble, V. Falaleev, E. Formenti, W. Funk, L. Gatignon, A. Gonidec, P. Grafstrom, B. Hallgren, P. Kapusta, G. Kesseler, W. Kubischta, W. Iwanski, A. Lacourt, G. Laverriere, G. Linser, C. Ljuslin, A. Marchioro, M. Mast, J. R. Matheys, M. Morel, A. Norton, J. P. Orlic, B. Panzer-Steindel, D. Schinzel, W. Seidl, H. Taureg, J. C. Tarle, M. Velasco, O. Vossnack, H. Wahl, R. Wertelaers, J. Weterings, C. Cheshkov, A. Gaponenko, E. Goudzovski, P. Hristov, A. Kalinin, V. Kekelidze, Y. Kozhevnikov, D. Madigozhin, N. Molokanova, Y. Potrebenikov, A. Tkatchev, A. Zinchenko, O. Boyle, I. Knowles, V. Martin, H. Parsons, K. J. Peach, R. Sacco, E. Veitch, A. Walker, V. Carassiti, M. Contalbrigo, A. C. Ramusino, R. Dalpiaz, C. Damiani, J. Duclos, P. Ferretti, P. L. Frabetti, A. Gianoli, M. Martini, E. Petrucci, M. Porcu, F. Rossi, M. Savrie, M. Scarpa, C. Simani, A. Bizzeti, M. Calvetti, G. Collazuol, G. Graziani, E. Lacopini, M. Lenti, 
F. Martelli, A. Michetti, G. Ruggiero, M. Veltri, H. G. Becker, M. Behler, H. Blumer, D. Coward, C. Ebersberger, K. Eppard, M. Eppard, H. Fox, K. H. Geib, A. Hirstius, A. Kalter, K. Kleinknecht, U. Koch, L. Kopke, R. L. da Silva, S. Luitz, P. Marouelli, L. Masetti, I. Melzer-Pellmann, U. Moosbrugger, C. Morales, A. Peters, B. Renk, J. Scheidt, J. Schmidt, S. A. Schmidt, V. Schonharting, Y. Schue, J. Staeck, R. Wanke, R. Wilhelm, A. Winhart, M. Wittgen, O. Zeitnitz, A. Dabrowski, T. M. F. Martin, J. C. Chollet, S. Crepe, C. De la Taille, L. Fayard, L. Iconomidou-Fayard, G. Martin-Chassard, J. Ocariz, G. Unal, I. Wingerter-Seez, G. Anzivino, E. Bordacchini, R. Cenci, E. Imbergamo, P. Lariccia, P. Lubrano, A. Mestvirishivili, A. Papi, M. Pepe, M. Piccini, M. Punturo, C. Talamonti, F. Tondini, L. Bertanza, P. Calafiura, R. Carosi, R. Casali, C. Cerri, M. Cirilli, E. Costantini, R. Fantechi, E. Fidecaro, L. Fiorini, S. Giudici, B. Gorini, F. Laico, G. Lamanna, I. Mannelli, V. Marzulli, D. Passuello, G. M. Pierazzini, F. Raffaelli, M. Sozzi, R. Tripiccione, S. Anvar, D. Bederede, E. Bugeon, J. B. Cheze, J. Cogan, M. De Beer, P. Debu, D. Durand, S. Edard, J. L. Fallou, A. Formica, L. Gosset, R. G. de Cassagnac, J. Heitzmann, H. Le Provost, F. Louis, I. Mandzhavidze, E. Mazzucato, A. Migliori, M. Mur, B. Peyaud, S. Schanne, O. Steinkamp, G. Tarte, R. Turlay, B. Vallage, M. Holder, I. Augustin, M. Bender, A. Maier, I. Schwarz, M. Ziolkowski, R. Arcidiacono, P. L. Barberis, F. Benotto, E. Bertolino, C. Biino, O. Brunasso, N. Cartiglia, M. Clemencic, D. Dattola, S. G. Lopez, G. Govi, R. Guida, F. Marchetto, E. Menichetti, S. Palestini, N. Pastrone, A. Chlopik, Z. Guzik, J. Nassalski, E. Rondio, M. Szleper, W. Wislicki, S. Wronka, H. Dibon, G. Fischer, M. Jeitler, M. Markytan, I. Mikulec, G. Neuhofer, M. Pernicka, A. Taurok, L. Widhalm, and N. A. Collaboration, The beam and detector for the na48 neutral kaon cp violation experiment at CERN, Nucl. Instrum. Methods Phys. Res. Sect. A 574, 433 (2007).

[9] A. M. Tarantin, E. N. Tsyganov, and S. A. Vorobiev, Computer-simulation of deflection effects for relativistic charged-particles in a curved crystal, Phys. Lett. A 72, 145 (1979).

[10] V. M. Biryukov, On the theory of proton-beam multiturn extraction with bent single-crystals, Nucl. Instrum. Methods Phys. Res. Sect. B 53, 202 (1991).

[11] J. Lindhard, Influence of crystal lattice on motion of energetic charged particles, Kongelige Danske Videnskabernes Selskab, 34, 14 (1965).

[12] A. G. Afonin, A. A. Arkhipenko, V. I. Baranov, V. M. Biryukov, Y. A. Chesnokov, V. A. Gavrilushkin, V. N. Gres, V. I. Kotov, V. A. Zelenov, B. A. Chunin, M. G. Gordeeva, Y. M. Ivanov, A. A. Petrunin, and V. V. Skorobogatov, Highefficiency multipass extraction of $70-\mathrm{GeV}$ protons from an accelerator with a short bent crystal, Phys. Lett. B 435, 240 (1998).

[13] S. Baricordi, V. Guidi, A. Mazzolari, D. Vincenzi, and M. Ferroni, Shaping of silicon crystals for channelling experiments through anisotropic chemical etching, J. Phys. D 41, 245501 (2008)

[14] W. Scandale, D. A. Still, A. Carnera, G. Della Mea, D. De Salvador, R. Milan, A. Vomiero, S. Baricordi, P. Dalpiaz, M. Fiorini, V. Guidi, G. Martinelli, A. Mazzolari, E. Milan, G. Ambrosi, P. Azzarello, R. Battiston, B. Bertucci, W. J. Burger,
M. Ionica, P. Zuccon, G. Cavoto, R. Santacesaria, P. Valente, E. Vallazza, A. G. Afonin, V. T. Baranov, Y. A. Chesnokov, V. I. Kotov, V. A. Maisheev, I. A. Yaznin, S. V. Afansiev, A. D. Kovalenko, A. M. Taratin, A. S. Denisov, Y. A. Gavrikov, Y. M. Ivanov, V. G. Ivochkin, S. V. Kosyanenko, A. A. Petrunin, V. V. Skorobogatov, V. M. Suvorov, D. Bolognini, L. Foggetta, S. Hasan, and M. Prest, High-Efficiency Volume Reflection of an Ultrarelativistic Proton Beam with a Bent Silicon Crystal, Phys. Rev. Lett. 98, 154801 (2007).

[15] W. Scandale, A. Vomiero, S. Baricordi, P. Dalpiaz, M. Fiorini, V. Guidi, A. Mazzolari, R. Milan, G. D Mea, G. Ambrosi, B. Bertucci, W. J. Burger, P. Zuccon, G. Cavoto, R. Santacesaria, P. Valente, E. Vallazza, A. G. Afonin, Y. A. Chesnokov, V. A. Maisheev, I. A. Yazynin, A. D. Kovalenkok, A. M. Taratin, A. S. Denisov, Y. A. Gavrikov, Y. M. Ivanov, L. P. Lapina, L. G. Malyarenko, V. V. Skorobogatov, V. M. Suvorov, S. A. Vavilov, D. Bolognini, S. Hasan, and M. Prest, Observation of nuclear dechanneling for high-energy protons in crystals, Phys. Lett. B 680, 129 (2009).

[16] W. Scandale, A. Vomiero, S. Baricordi, P. Dalpiaz, M. Fiorini, V. Guidi, A. Mazzolari, G. D Mea, R. Milan, G. Ambrosi, P. Zuccon, B. Bertucci, W. Burger, M. Duranti, G. Cavoto, R. Santacesaria, P. Valente, C. Luci, F. Iacoangeli, E. Vallazza, A. G. Afonin, Y. A. Chesnokov, V. I. Kotov, V. A. Maisheev, I. A. Yazynin, A. D. Kovalenko, A. M. Taratin, A. S. Denisov, Y. A. Gavrikov, Y. M. Ivanov, L. P. Lapina, L. G. Malyarenko, V. V. Skorogobogatov, V. M. Suvorov, S. A. Vavilov, D. Bolognini, S. Hasan, A. Mozzanica, and M. Prest, Observation of Multiple Volume Reflection of Ultrarelativistic Protons by a Sequence of Several Bent Silicon Crystals, Phys. Rev. Lett. 102, 084801 (2009).

[17] V. Carassiti, P. Dalpiaz, V. Guidi, A. Mazzolari, and M. Melchiorri, Note: Rigid holder to host and bend a crystal for multiple volume reflection of a particle beam, Rev. Sci. Instrum. 81, 066106 (2010).

[18] W. Scandale, G. Arduini, M. Butcher, F. Cerutti, M. Garattini, S. Gilardoni, L. Lari, A. Lechner, R. Losito, A. Masi, A. Mereghetti, E. Metral, D. Mirarchi, S. Montesano, S. Redaelli, R. Rossi, P. Schoofs, G. Smirnov, E. Bagli, L. Bandiera, S. Baricordi, P. Dalpiaz, G. Germogli, V. Guidi, A. Mazzolari, D. Vincenzi, G. Claps, S. Dabagov, D. Hampai, F. Murtas, G. Cavoto, F. Iacoangeli, L. Ludovici, R. Santacesaria, P. Valente, F. Galluccio, A. G. Afonin, Y. A. Chesnokov, V. A. Maisheev, Y. E. Sandomirskiy, A. A. Yanovich, A. Yazynin, A. D. Kovalenko, A. M. Taratin, Y. A. Gavrikov, Y. M. Ivanov, L. P. Lapina, W. Ferguson, J. Fulcher, G. Hall, M. Pesaresi, M. Raymond, D. Bolognini, S. Hasan, M. Prest, and E. Vallazza, Observation of nuclear dechanneling length reduction for high energy protons in a short bent crystal, Phys. Lett. B 743, 440 (2015).

[19] J. Bak, G. Melchart, E. Uggerhoj, J. S. Forster, P. R. Jensen, H. Madsboll, S. P. Moller, H. Nielsen, G. Petersen, H. Schiott, J. J. Grob, and P. Siffert, Bending of high-energy beams using axial and planar channeling, Phys. Lett. B 93, 505 (1980).

[20] W. Scandale, A. Vomiero, S. Baricordi, P. Dalpiaz, M. Fiorini, V. Guidi, A. Mazzolari, G. D Mea, R. Milan, G. Ambrosi, B. Bertucci, W. J. Burger, P. Zuccon, G. Cavoto, C. Luci, R. Santacesaria, P. Valente, E. Vallazza, A. G. Afonin, Y. A. Chesnokov, V. A. Maisheev, I. A. Yazynin, A. D. Kovalenko, A. M. Taratin, A. S. Denisov, Y. A. Gavrikov, Y. M. Ivanov, 
L. P. Lapina, L. G. Malyarenko, V. V. Skorobogatov, V. M. Suvorov, S. A. Vavilov, D. Bolognini, S. Hasan, A. Mozzanica, and M. Prest, High-Efficiency Deflection of HighEnergy Protons through Axial Channeling in a Bent Crystal, Phys. Rev. Lett. 101, 164801 (2008).

[21] W. Scandale, A. Vomiero, E. Bagli, S. Baricordi, P. Dalpiaz, M. Fiorini, V. Guidi, A. Mazzolari, D. Vincenzi, R. Milan, G. D Mea, E. Vallazza, A. G. Afonin, Y. A. Chesnokov, V. A. Maisheev, I. A. Yazynin, V. M. Golovatyuk, A. D. Kovalenko, A. M. Taratin, A. S. Denisov, Y. A. Gavrikov, Y. M. Ivanov, L. P. Lapina, L. G. Malyarenko, V. V. Skorobogatov, V. M. Suvorov, S. A. Vavilov, D. Bolognini, S. Hasan, A. Mattera, M. Prest, and V. V. Tikhomirov, First observation of multiple volume reflection by different planes in one bent silicon crystal for high-energy protons, Phys. Lett. B 682, 274 (2009).

[22] W. Scandale, A. Vomiero, E. Bagli, S. Baricordi, P. Dalpiaz, M. Fiorini, V. Guidi, A. Mazzolari, D. Vincenzi, R. Milan, G. D Mea, E. Vallazza, A. G. Afonin, Y. A. Chesnokov, V. A. Maisheev, I. A. Yazynin, V. M. Golovatyukh, A. D. Kovalenko, A. M. Taratin, A. S. Denisov, Y. A. Gavrikov, Y. M. Ivanov, L. P. Lapina, L. G. Malyarenko, V. V. Skorobogatov, V. M. Suvorov, S. A. Vavilov, D. Bolognini, S. Hasan, and M. Prest, Deflection of high-energy negative particles in a bent crystal through axial channeling and multiple volume reflection stimulated by doughnut scattering, Phys. Lett. B 693, 545 (2010).

[23] V. Guidi, A. Mazzolari, and V. Tikhomirov, On the observation of multiple volume reflection from different planes inside one bent crystal, J. Appl. Phys. 107, 114908 (2010).

[24] L. Bandiera, A. Mazzolari, E. Bagli, G. Germogli, V. Guidi, A. Sytov, I. V. Kirillin, N. F. Shul'ga, A. Berra, D. Lietti, M. Prest, D. De Salvador, and E. Vallazza, Relaxation of axially confined $400 \mathrm{GeV} / \mathrm{c}$ protons to planar channeling in a bent crystal, Eur. Phys. J. C 76, 1 (2016).

[25] Y. M. Ivanov, A. A. Petrunin, and V. V. Skorobogatov, Observation of the elastic quasi-mosaicity effect in bent silicon single crystals, JETP Lett. 81, 99 (2005).

[26] A. G. Afonin, V. T. Baranov, V. M. Biryukov, M. B. H. Breese, V. N. Chepegin, Y. A. Chesnokov, V. Guidi, Y. M. Ivanov, V. I. Kotov, G. Martinelli, W. Scandale, M. Stefancich, V. I. Terekhov, D. Trbojevic, E. F. Troyanov, and D. Vincenzi, High-Eefficiency Beam Extraction and Collimation Using Channeling in Very Short Bent Crystals, Phys. Rev. Lett. 87, 094802 (2001).

[27] S. Baricordi, V. Guidi, A. Mazzolari, G. Martinelli, A. Carnera, D. De Salvador, A. Sambo, G. Della Mea, R. Milan, A. Vomiero, and W. Scandale, Optimal crystal surface for efficient channeling in the new generation of hadron machines, Appl. Phys. Lett. 91, 061908 (2007).

[28] D. Mirarchi, V. Avati, R. Bruce, M. Butcher, M. D’Andrea, M. Di Castro, M. Deile, B. Dziedzic, K. Hiller, S. Jakobsen, J. Kašpar, K. Korcyl, I. Lamas, A. Masi, A. Mereghetti, H. G. Morales, Y. Gavrikov, S. Redaelli, B. S. Ferrando, P. Serrano, M. S. Camillocci, and N. Turini, Reducing beam-related background on forward physics detectors using crystal collimation at the Large Hadron Collider, Phys. Rev. Appl. 14, 064066 (2020).

[29] W. Scandale, G. Arduini, M. Butcher, F. Cerutti, M. Garattini, S. Gilardoni, A. Lechner, R. Losito, A. Masi, A. Mereghetti, E. Metral, D. Mirarchi, S. Montesano, S. Redaelli, R. Rossi,
P. Schoofs, G. Smirnov, E. Bagli, L. Bandiera, S. Baricordi, P. Dalpiaz, G. Germogli, V. Guidi, A. Mazzolari, D. Vincenzi, G. Claps, S. Dabagov, D. Hampai, F. Murtas, G. Cavoto, F. Iacoangeli, L. Ludovici, R. Santacesaria, P. Valente, F. Galluccio, A. G. Afonin, Y. A. Chesnokov, A. A. Durum, V. A. Maisheev, Y. E. Sandomirskiy, A. A. Yanovich, A. D. Kovalenko, A. M. Taratin, Y. A. Gavrikov, Y. M. Ivanov, L. P. Lapina, J. Fulcher, G. Hall, M. Pesaresi, and M. Raymond, Observation of strong leakage reduction in crystal assisted collimation of the SPS beam, Phys. Lett. B 748, 451 (2015).

[30] W. Scandale, G. Arduini, R. Assmann, C. Bracco, F. Cerutti, J. Christiansen, S. Gilardoni, E. Laface, R. Losito, A. Masi, E. Metral, D. Mirarchi, S. Montesano, V. Previtali, S. Redaelli, G. Valentino, P. Schoofs, G. Smirnov, L. Tlustos, E. Bagli, S. Baricordi, P. Dalpiaz, V. Guidi, A. Mazzolari, D. Vincenzi, S. Dabagov, F. Murtas, A. Carnera, G. Della Mea, D. De Salvador, A. Lombardi, O. Lytovchenko, M. Tonezzer, G. Cavoto, L. Ludovici, R. Santacesaria, P. Valente, F. Galluccio, A. G. Afonin, M. K. Bulgakov, Y. A. Chesnokov, V. A. Maisheev, I. A. Yazynin, A. D. Kovalenko, A. M. Taratin, V. V. Uzhinskiy, Y. A. Gavrikov, Y. M. Ivanov, L. P. Lapina, V. V. Skorobogatov, W. Ferguson, J. Fulcher, G. Hall, M. Pesaresi, M. Raymond, A. Rose, M. Ryan, O. Zorba, G. RobertDemolaize, T. Markiewicz, M. Oriunno, and U. Wienands, Comparative results on collimation of the SPS beam of protons and $\mathrm{Pb}$ ions with bent crystals, Phys. Lett. B 703, 547 (2011).

[31] W. Scandale, G. Arduini, R. Assmann, C. Bracco, S. Gilardoni, V. Ippolito, E. Laface, R. Losito, A. Masi, E. Metral, V. Previtali, S. Redaelli, M. Silari, L. Tlustos, E. Bagli, S. Baricordi, P. Dalpiaz, V. Guidi, A. Mazzolari, D. Vincenzi, G. Della Mea, A. Lombardi, D. De Salvador, E. Vallazza, D. Bolognini, S. Hasan, D. Lietti, V. Mascagna, A. Mattera, M. Prest, G. Cavoto, L. Ludovici, D. Mirarchi, R. Santacesaria, P. Valente, F. Murtas, A. G. Afonin, Y. A. Chesnokov, V. A. Maisheev, I. A. Yazynin, A. D. Kovalenko, A. M. Taratin, A. S. Denisov, Y. A. Gavrikov, Y. M. Ivanov, L. P. Lapina, L. G. Malyarenko, V. V. Skorobogatov, V. M. Suvorov, S. A. Vavilov, N. Mokhov, D. Still, G. Robert-Demolaize, T. Markiewicz, and M. Oriunno, First results on the sps beam collimation with bent crystals, Phys. Lett. B 692, 78 (2010).

[32] I. B. A. G. Apollinari, O. Brüning, M. Lamont, and L. Rossi, eds., High-Luminosity Large Hadron Collider (HL-LHC), Preliminary Design Report (CERN, Geneva, 2015).

[33] T. Whyntie, The high luminosity large hadron collider: The new machine for illuminating the mysteries of universe in Contemporary Physics, edited by O. Brüning and L. Rossi (Taylor \& Francis, London, 2017).

[34] E. Uggerhoj and U. I. Uggerhoi, Strong crystalline fieldsA possibility for extraction from the LHC, Nucl. Instrum. Methods Phys. Res. Sect. B 234, 31 (2005).

[35] D. Kikola, S. J. Brodsky, G. Cavoto, C. Da Silva, F. Donato, M. G. Echevarria, E. G. Ferreiro, C. Hadjidakis, I. Hrivnacova, A. Klein, A. Kurepin, A. Kusina, J. P. Lansberg, C. Lorce, F. Lyonnet, Y. Makdisi, L. Massacrier, S. Porteboeuf, C. Quintans, A. Rakotozafindrabe, P. Robbe, W. Scandale, I. Schienbein, J. Seixas, H. S. Shao, A. Signori, N. Topilskaya, B. Trzeciak, A. Uras, J. Wagner, N. Yamanaka, Z. Yang, and A. Zelenski, A fixed-target programme at the LHC for heavyion, hadron, spin and astroparticle physics: AFTER@LHC, Nucl. Phys. A 982, 971 (2019). 
[36] C. Barschelet al., Report from the LHC Fixed Target Working Group of the CERN Physics Beyond Colliders Forum (CERN, Geneva, 2019).

[37] D. Mirarchi, A. S. Fomin, S. Redaelli, and W. Scandale, Layouts for fixed-target experiments and dipole moment measurements of short-living baryons using bent crystals at the LHC, Eur. Phys. J. C 80, 929 (2020).

[38] V. Biryukov, Computer-Simulation of Crystal Extraction of Protons from a Large-Hadron-Collider Beam, Phys. Rev. Lett. 74, 2471 (1995).

[39] V. G. Baryshevsky, The possibility to measure the magnetic moments of short-lived particles (charm and beauty baryons) at LHC and FCC energies using the phenomenon of spin rotation in crystals, Phys. Lett. B 757, 426 (2016).

[40] E. Bagli, L. Bandiera, G. Cavoto, V. Guidi, L. Henry, D. Marangotto, F. M. Vidal, A. Mazzolari, A. Merli, N. Neri, and J. R. Vidal, Electromagnetic dipole moments of charged baryons with bent crystals at the LHC, Eur. Phys. J. C 77, 19 (2017).

[41] V. G. Baryshevsky, Spin rotation and depolarization of highenergy particles in crystals at LHC and FCC energies. The possibility to measure the anomalous magnetic moments of short-lived particles and quadrupole moment of Omegahyperon, Nucl. Instrum. Methods Phys. Res. Sect. B 402, 5 (2017).

[42] F. J. Botella, L. M. G. Martin, D. Marangotto, F. M. Vidal, A. Merli, N. Neri, A. Oyanguren, and J. R. Vidal, On the search for the electric dipole moment of strange and charm baryons at lhc, Eur. Phys. J. C 77, 181 (2017).

[43] A. S. Fomin, A. Y. Korchin, A. Stocchi, O. A. Bezshyyko, L. Burmistrov, S. P. Fomin, I. V. Kirillin, L. Massacrier, A. Natochii, P. Robbe, W. Scandale, and N. F. Shul'ga, Feasibility of measuring the magnetic dipole moments of the charm baryons at the LHC using bent crystals, J. High Energy Phys. 08 (2017) 120.

[44] A. S. Fomin, S. Barsuk, A. Y. Korchin, E. Kou, V. A. Kovalchuk, M. Liul, A. Natochii, E. Niel, P. Robbe, and A. Stocchi, The prospect of charm quark magnetic moment determination, Eur. Phys. J. C 80, 358 (2020).

[45] C. Hadjidakis, D. Kikoła, J. P. Lansberg, L. Massacrier, M. G. Echevarria, A. Kusina, I. Schienbein, J. Seixas, H. S. Shao, A. Signori, B. Trzeciak, S. J. Brodsky, G. Cavoto, C. D. Silva, F. Donato, E. G. Ferreiro, I. Hrivnacova, A. Klein, A. Kurepin, F. Lyonnet, Y. Makdisi, C. Lorcé, S. Porteboeuf, C. Quintans, A. Rakotozafindrabe, P. Robbe, N. Topilskaya, A. Uras, J. Wagner, N. Yamanaka, Z. Yang, and A. Zelenski, A fixed-target programme at the LHC: physics case and projected performances for heavy-ion, hadron, Spin Astroparticle Studies, arXiv:1807.00603 (2018).

[46] F. Galluccio, C. Hadjidakis, A. Kurepin, L. Massacrier, S. Porteboeuf, K. Pressard, W. Scandale, N. Topilskaya, B. Trzeciak, A. Uras, and D. Kikola, Physics opportunities for a fixed-target programme in the ALICE experiment, https://indico.cern.ch/event/765096/contributions/3295620/ (2019).

[47] A. Mazzolari, M. Romagnoni, R. Camattari, E. Bagli, L. Bandiera, G. Germogli, V. Guidi, and G. Cavoto, Bent crystals for efficient beam steering of multi TeV-particle beams, Eur. Phys. J. C 78, 720 (2018).
[48] F. M. Velotti, L. S. Esposito, M. A. Fraser, V. Kain, S. Gilardoni, B. Goddard, M. Pari, J. Prieto, R. Rossi, W. Scandale, L. S. Stoel, F. Galluccio, M. Garattini, and Y. Gavrikov, Septum shadowing by means of a bent crystal to reduce slow extraction beam loss, Phys. Rev. Accel. Beams 22, 093502 (2019).

[49] M. Garattini, Sherpa: "slow high-efficiency extraction from ring positron accelerator" (2020), available from: https:// agenda.infn.it/event/21911/.

[50] A. V. Korol, A. V. Solov'yov, and W. Greiner, Channeling and radiation in periodically bent crystals, in Springer Series on Atomic, Optical, and Plasma Physics (Springer, Berlin 2014).

[51] A. Kostyuk, Crystalline Undulator with a Small Amplitude and a Short Period, Phys. Rev. Lett. 110, 115503 (2013).

[52] C. H. Xu, R. Chehab, P. Sievers, X. Artru, M. Chevallier, O. Dadoun, G. X. Pei, V. M. Strakhovenko, and A. Variola, A positron source using an axially oriented crystal associated to a granular amorphous converter, Chin. Phys. C 36, 871 (2012).

[53] L. Bandiera, V. Haurylavets, and V. Tikhomirov, Compact electromagnetic calorimeters based on oriented scintillator crystals, Nucl. Instrum. Methods Phys. Res. Sect. A 936, 124 (2019).

[54] L. Bandiera, V. V. Tikhomirov, M. Romagnoni, N. Argiolas, E. Bagli, G. Ballerini, A. Berra, C. Brizzolari, R. Camattari, D. De Salvador, V. Haurylavets, V. Mascagna, A. Mazzolari, M. Prest, M. Soldani, A. Sytov, and E. Vallazza, Strong Reduction of the Effective Radiation Length in an Axially Oriented Scintillator Crystal, Phys. Rev. Lett. 121, 021603 (2018).

[55] A. D. Kovalenko, W. Scandale, and A. M. Taratin, Bent crystal extraction from a $100 \mathrm{TeV}$ proton collider, Nucl. Instrum. Methods Phys. Res. Sect. B 355, 390 (2015).

[56] A. I. Sytov, V. V. Tikhomirov, and A. S. Lobko, Crystal collimator systems for high energy frontier, Phys. Rev. Accel. Beams 20, 071001 (2017).

[57] M. Benedikt, A. Blondel, P. Janot, M. Klein, M. Mangano, M. McCullough, V. Mertens, K. Oide, W. Riegler, D. Schulte, and F. Zimmermann, Future circular colliders, Annu. Rev. Nucl. Part. Sci. 69, 389 (2019).

[58] A. Seryi, Future prospects of accelerator science for particle physics, Nucl. Instrum. Methods Phys. Res. Sect. A 623, 23 (2010).

[59] L. Rinolfi, R. Chehab, O. Dadoun, T. Kamitani, V. Strakhovenko, and A. Variola, Brilliant positron sources for CLIC and other collider projects, Nucl. Instrum. Methods Phys. Res. Sect. B 309, 50 (2013).

[60] X. Lou, The Circular Electron Positron Collider, Nature Reviews Physics 1, 232 (2019).

[61] S. A. Bogacz, D. B. Cline, and D. A. Sanders, A mu(+)mu(-) crystal collider concept, Nucl. Instrum. Methods Phys. Res. Sect. B 119, 199 (1996).

[62] E. S. F. P. P. P. Group, Physics briefing book, arXiv:1910.11775.

[63] D. Mirarchi, G. Hall, S. Redaelli, and W. Scandale, Design and implementation of a crystal collimation test stand at the Large Hadron Collider, Eur. Phys. J. C 77, 424 (2017).

[64] V. M. Biryukov, V. N. Chepegin, Y. A. Chesnokov, V. Guidi, and W. Scandale, Crystal collimation as an option for the large hadron colliders, Nucl. Instrum. Methods Phys. Res. Sect. B 234, 23 (2005). 
[65] R. W. Assmann, O. Aberle, G. Bellodi, A. Bertarelli, C. Bracco, H. Braun, M. Brugger, S. Calatroni, R. Chamizo, A. Dallocchio, B. Dehning, A. Ferrari, P. Gander, A. Grudiev, E. B. Holzer, J. B. Jeanneret, J. M. Jiménez, M. Jonker, Y. Kadi, K. Kershaw, J. Lendaro, J. Lettry, R. Losito, M. Magistris, A. Masi, M. Mayer, E. Métral, R. Perret, C. Rathjen, S. Redaelli, G. Robert-Démolaize, S. Roesler, F. Ruggiero, M. Santana, P. Sievers, M. Sobczak, A. Tsoulou, V. Vlachoudis, T. Weiler, I. S. Baishev, and I. Kurochkin, The final collimation system for the LHC, in LHC-PROJECT (CERN, Geneva, 2006).

[66] S. Redaelli, ICFA Mini-Workshop on Tracking for Collimation in Particle Accelerators (CERN, Geneva, 2015).

[67] H. Burkhardt, B. Goddard, Y. Kadi, V. Kain, T. Risselada, and W. Weterings, Collimation in the transfer lines to the LHC, 2005 IEEE Particle Accelerator Conference (IEEE, New York, 2005), pp. 2825-2827.

[68] A. Drees, R. Fliller, and W. Fu, RHIC loss limitations and collimation, in High Intensity and High Brightness Hadron Beams, edited by I. Hofmann, R. W. Hasse, and J. M. Lagniel(2005), pp. 55-59.

[69] T. Wei, Q. B. Wu, and W. Q. Li, Beam collimation for CSNS/RCS, Chin. Phys. C 34, 506 (2010).

[70] R. Bruce, R. W. Assmann, V. Boccone, C. Bracco, M. Brugger, M. Cauchi, F. Cerutti, D. Deboy, A. Ferrari, L. Lari, A. Marsili, A. Mereghetti, D. Mirarchi, E. Quaranta, S. Redaelli, G. Robert-Demolaize, A. Rossi, B. Salvachua, E. Skordis, C. Tambasco, G. Valentino, T. Weiler, V. Vlachoudis, and D. Wollmann, Simulations and measurements of beam loss patterns at the cern large hadron collider, Phys. Rev. Special Topics 17, 081004 (2014).

[71] I. Strasik, I. Prokhorov, and O. Boine-Frankenheim, Beam halo collimation in heavy ion synchrotrons, Phys. Rev. Special Topics 18, 081001 (2015).

[72] The High Luminosity Large Hadron Collider The New Machine for Illuminating the Mysteries of Universe (World Scientific, Singapore, 2015).

[73] A. Gorzawski, A. Abramov, R. Bruce, N. Fuster-Martinez, M. Krasny, J. Molson, S. Redaelli, and M. Schaumann, Collimation of partially stripped ions in the cern Large Hadron Collider, Phys. Rev. Accel. Beams 23, 101002 (2020).

[74] N. Fuster-Martínez, R. Bruce, F. Cerutti, R. De Maria, P. Hermes, A. Lechner, A. Mereghetti, J. Molson, S. Redaelli, E. Skordis, A. Abramov, and L. Nevay, Simulations of heavyion halo collimation at the CERN Large Hadron Collider: Benchmark with measurements and cleaning performance evaluation, Phys. Rev. Accel. Beams 23, 111002 (2020).

[75] G. Apollinari, I. Béjar Alonso, O. Brüning, P. Fessia, M. Lamont, L. Rossi, and L. Tavian, High-Luminosity Large Hadron Collider (HL-LHC), Technical Design Report V. 0.1. (CERN, Geneva, 2017).

[76] R. P. Fliller, A. Drees, D. Gassner, L. Hammons, G. McIntyre, S. Peggs, D. Trbojevic, V. Biryukov, Y. Chesnokov, and V. Terekhov, Rhic crystal collimation, Nucl. Instrum. Methods Phys. Res. Sect. B 234, 47 (2005).

[77] W. Scandale, G. Arduini, M. Butcher, F. Cerutti, M. Garattini, S. Gilardoni, A. Lechner, R. Losito, A. Masi, D. Mirarchi, S. Montesano, S. Redaelli, R. Rossi, P. Schoofs, G. Smirnov, G. Valentino, D. Breton, L. Burmistrov, V. Chaumat, S. Dubos, J. Maalmi, V. Puill, A. Stocchi, E. Bagli, L. Bandiera, G. Germogli, V. Guidi, A. Mazzolari, S. Dabagov, F. Murtas,
F. Addesa, G. Cavoto, F. Iacoangeli, F. Galluccio, A. G. Afonin, Y. A. Chesnokov, A. A. Durum, V. A. Maisheev, Y. E. Sandomirskiy, A. A. Yanovich, A. D. Kovalenko, A. M. Taratin, A. S. Denisov, Y. A. Gavrikov, Y. M. Ivanov, L. P. Lapina, L. G. Malyarenko, V. V. Skorobogatov, T. James, G. Hall, M. Pesaresi, and M. Raymond, Measurements of multiple scattering of high energy protons in bent silicon crystals, Nucl. Instrum. Methods Phys. Res. Sect. B 402, 291 (2017).

[78] W. Scandale, M. Calviani, M. D'Andrea, L. S. Esposito, M. Garattini, S. Gilardoni, S. Montesano, A. Natochii, R. Rossi, G. I. Smirnov, C. L. T. Martin, V. Zhovkovska, E. Bagli, L. Bandiera, V. Guidi, A. Mazzolari, M. Romagnoni, F. Murtas, F. Galluccio, F. Addesa, F. Iacoangeli, A. D. Kovalenko, A. M. Taratin, Y. A. Gavrikov, Y. M. Ivanov, M. A. Koznov, M. V. Malkov, L. G. Malyarenko, I. G. Mamunct, J. Borg, T. James, G. Hall, and M. Pesaresi, Beam steering performance of bent silicon crystals irradiated with high-intensity and high-energy protons, Eur. Phys. J. C 79, 933 (2019).

[79] G. Arduini, C. Biino, M. Clement, K. Cornelis, N. Doble, K. Elsener, G. Ferioli, G. Fidecaro, L. Gatignon, P. Grafstrom, M. Gyr, W. Herr, J. Klem, U. Mikkelsen, E. Weisse, S. P. Moller, E. Uggerhoj, A. Taratin, A. Freund, P. Keppler, and J. Major, Deflection and Extraction of $\mathrm{Pb}$ Ions up to $33 \mathrm{TeV} / \mathrm{c}$ by a Bent Silicon Crystal, Phys. Rev. Lett. 79, 4182 (1997).

[80] W. Scandale, F. Andrisani, G. Arduini, F. Cerutti, M. Garattini, S. Gilardoni, A. Masi, D. Mirarchi, S. Montesano, S. Petrucci, S. Redaelli, P. Schoofs, R. Rossi, D. Breton, D. Chaumat, S. Dubos, J. Maalmi, A. Natochii, V. Puill, A. Stocchi, E. Bagli, L. Bandiera, G. Germogli, V. Guidi, A. Mazzolari, F. Murtas, F. Addesa, G. Cavoto, F. Iacoangeli, F. Galluccio, A. G. Afonin, Y. A. Chesnokov, A. A. Durum, V. A. Maisheev, Y. E. Sandomirskiy, A. A. Yanovich, A. D. Kovalenko, A. M. Taratin, G. I. Smirnov, A. S. Desinov, Y. A. Gavrikov, Y. M. Ivanov, L. P. Lapina, L. G. Malyarenko, V. V. Skorobogatov, J. Fulcher, T. James, G. Hall, M. Pesaresi, and M. Raymond, Study of inelastic nuclear interactions of $400 \mathrm{GeV} / \mathrm{c}$ protons in bent silicon crystals for beam steering purposes, Eur. Phys. J. C 78, 505 (2018).

[81] W. Scandale, A. M. Taratin, and A. D. Kovalenko, Experiments with crystal deflectors for high energy ion beams: Electromagnetic dissociation probability for well channeled ions, Phys. Rev. Special Topics Accel. Beams 16, 011001 (2013).

[82] U. I. Uggerhoj, H. D. Hansen, K. Jessen, H. Knudsen, E. Uggerhoj, C. Scheidenberger, C. Biino, M. Clement, N. Doble, K. Elsener, L. Gatignon, P. Grafstrom, P. Sona, A. Mangiarotti, and S. Ballestrero, Strong suppression of nuclear-charge changing interactions for $18 \mathrm{TeV} / \mathrm{c}$ In ions channeled through a bent Si crystal, Phys. Lett. B 619, 240 (2005).

[83] V. Biryukov, Computer-simulation of dislocation dechanneling in bent crystals at tera-electron-volt energies, Phys. Rev. E 52, 2045 (1995).

[84] E. Sirtl and A. Adler, Chromic-hydrofluoric acid as a specific system for the development of etch pits on silicon, Z. Metal. 52, 529 (1961).

[85] F. S. D. Aragona, J. Electrochem. Soc. 119, 948 (1972).

[86] B. K. Tanner, Crystal assessment by x-ray topography using synchrotron radiation, Prog. Cryst. Growth Charact. 1, 23 (1977). 
[87] K. Elsener, G. Fidecaro, M. Gyr, W. Herr, J. Klem, U. Mikkelsen, S. P. Moller, E. Uggerhoj, G. Vuagnin, and E. Weisse, Proton extraction from the cern sps using bent silicon crystals, Nucl. Instrum. Methods Phys. Res. Sect. B 119, 215 (1996).

[88] W. Scandale, G. Arduini, R. Assmann, F. Cerutti, S. Gilardoni, E. Laface, R. Losito, A. Masi, E. Metral, D. Mirarchi, S. Montesano, V. Previtali, S. Redaelli, G. Valentino, P. Schoofs, G. Smirnov, E. Bagli, S. Baricordi, P. Dalpiaz, V. Guidi, A. Mazzolari, D. Vincenzi, S. Dabagov, F. Murtas, G. Claps, G. Cavoto, F. Iacoangeli, L. Ludovici, R. Santacesaria, P. Valente, F. Galluccio, A. G. Afonin, M. K. Bulgakov, Y. A. Chesnokov, V. A. Maisheev, I. A. Yazynin, A. D. Kovalenko, A. M. Taratin, V. V. Uzhinskiy, Y. A. Gavrikov, Y. M. Ivanov, L. P. Lapina, V. V. Skorobogatov, W. Ferguson, J. Fulcher, G. Hall, M. Pesaresi, M. Raymond, A. Rose, M. Ryan, G. RobertDemolaize, T. Markiewicz, M. Oriunno, and U. Wienands, Strong reduction of the off-momentum halo in crystal assisted collimation of the SPS beam, Phys. Lett. B 714, 231 (2012).

[89] S. D. Jacobs, D. Golini, Y. Hsu, B. E. Puchebner, D. Strafford, W. I. Kordonski, I. V. Prokhorov, E. Fess, D. Pietrowski, and V. W. Kordonski, Magnetorheological finishing: A deterministic process for optics manufacturing, Int. Conf. Opt. Fabrication Testing 2576, 372 (1995).

[90] http://qedmrf.com/.

[91] S. D. Jacobs, S. A. Arrasmith, I. A. Kozhinova, L. L. Gregg, A. B. Shorey, H. J. Romanofsky, D. Golini, W. I. Kordonski, P. Dumas, and S. Hogan, An overview of magnetorheological finishing (MRF) for precision optics manufacturing, in Finishing of Advanced Ceramics and Glasses, edited by R. Sabia, V. A. Greenhut, and C. G. Pantano (1999), 185-199.

[92] C. L. Miao, S. N. Shaffir, J. C. Lambropoulos, J. Mici, and S. D. Jacobs, Shear stress in magnetorheological finishing for glasses, Appl. Opt. 48, 2585 (2009).

[93] J. C. Lambropoulos, C. L. Miao, and S. D. Jacobs, Magnetic field effects on shear and normal stresses in magnetorheological finishing, Opt. Express 18, 19713 (2010).

[94] G. Germogli, A. Mazzolari, V. Guidi, and M. Romagnoni, Bent silicon strip crystals for high-energy charged particle beam collimation, Nucl. Instrum. Methods Phys. Res. Sect. B 402, 308 (2017).

[95] A. S. Fomin, A. Y. Korchin, A. Stocchi, S. Barsuk, and P. Robbe, Feasibility of $\tau$-lepton electromagnetic dipole moments measurement using bent crystal at the LHC, J. High Energy Phys. (2019) 156.

[96] J. Fu, M. A. Giorgi, L. Henry, D. Marangotto, F. M. Vidal, A. Merli, N. Neri, and J. R. Vidal, Novel Method for the Direct Measurement of the tau Lepton Dipole Moments, Phys. Rev. Lett. 123, 011801 (2019).

[97] V. Guidi, L. Lanzoni, and A. Mazzolari, Patterning and modeling of mechanically bent silicon plates deformed through coactive stresses, Thin Solid Films 520, 1074 (2011).

[98] V. Bellucci, R. Camattari, V. Guidi, A. Mazzolari, G. Paterno, G. Mattei, C. Scian, and L. Lanzoni, Ion implantation for manufacturing bent and periodically bent crystals, Appl. Phys. Lett. 107, 064102 (2015).

[99] R. Camattari, G. Paterno, M. Romagnoni, V. Bellucci, A. Mazzolari, and V. Guidi, Homogeneous self-standing curved monocrystals, obtained using sandblasting, to be used as ma- nipulators of hard x-rays and charged particle beams, J. Appl. Crystallogr. 50, 145 (2017).

[100] R. Camattari, M. Romagnoni, L. Bandiera, E. Bagli, A. Mazzolari, A. Sytov, S. Haaga, M. Kabukcuoglu, S. Bode, D. Hanschke, A. Danilewsky, T. Baumbach, V. Bellucci, V. Guidi, and G. Cavoto, X-ray characterization of self-standing bent Si crystal plates for Large Hadron Collider beam extraction, J. Appl. Crystallogr. 53, 486 (2020).

[101] C. Ferrari, E. Buffagni, E. Bonnini, and D. Korytar, High diffraction efficiency in crystals curved by surface damage, J. Appl. Crystallogr. 46, 1576 (2013).

[102] E. Bagli, L. Bandiera, V. Bellucci, A. Berra, R. Camattari, D. De Salvador, G. Germogli, V. Guidi, L. Lanzoni, D. Lietti, A. Mazzolari, M. Prest, V. V. Tikhomirov, and E. Vallazza, Experimental evidence of planar channeling in a periodically bent crystal, Eur. Phys. J. C 74, 7 (2014).

[103] V. Bellucci, R. Camattari, V. Guidi, and A. Mazzolari, Bending of silicon plate crystals through superficial grooving: Modeling and experimentation, Thin Solid Films 520, 1069 (2011).

[104] S. G. Lekhnitskii, ed., Theory of Elasticity of an Anisotropic Body (Mir, Paris, 1981).

[105] R. Camattari, V. Guidi, V. Bellucci, and A. Mazzolari, The 'quasi-mosaic' effect in crystals and its applications in modern physics, J. Appl. Crystallogr. 48, 977 (2015).

[106] V. Bellucci, R. Camattari, G. Paterno, V. Guidi, and A. Mazzolari, Origin of quasi-mosaic effect for symmetric skew planes in a silicon or germanium plate, J. Appl. Crystallogr. 49, 1810 (2016).

[107] A. G. Afonin, V. T. Baranov, M. K. Bulgakov, I. S. Voinov, V. B. Ganenko, V. N. Gorlov, I. V. Ivanova, I. V. Kirillin, V. A. Maisheev, S. F. Reshetnikov, D. A. Savin, E. A. Syshchikov, V. I. Terekhov, V. I. Truten, Y. A. Chesnokov, P. N. Chirkov, N. F. Shul'ga, and I. A. Yazynin, A study of collimation and extraction of the U-70 accelerator beam using an axially oriented crystal, Instrum. Exper. Techniques 59, 196 (2016).

[108] W. Scandale, G. Arduini, M. Butcher, F. Cerutti, S. Gilardoni, A. Lechner, R. Losito, A. Masi, E. Metral, D. Mirarchi, S. Montesano, S. Redaelli, G. Smirnov, E. Bagli, L. Bandiera, S. Baricordi, P. Dalpiaz, G. Germogli, V. Guidi, A. Mazzolari, D. Vincenzi, G. Claps, S. Dabagov, D. Hampai, F. Murtas, G. Cavoto, M. Garattini, F. Iacoangeli, L. Ludovici, R. Santacesaria, P. Valente, F. Galluccio, A. G. Afonin, Y. A. Chesnokov, P. N. Chirkov, V. A. Maisheev, Y. E. Sandomirskiy, I. A. Yazynin, A. D. Kovalenko, A. M. Taratin, Y. A. Gavrikov, Y. M. Ivanov, L. P. Lapina, W. Ferguson, J. Fulcher, G. Hall, M. Pesaresi, and M. Raymond, Mirroring of $400 \mathrm{GeV} / \mathrm{c}$ protons by an ultra-thin straight crystal, Phys. Lett. B 734, 1 (2014).

[109] V. Guidi, A. Mazzolari, D. De Salvador, and L. Bacci, Deflection of MeV Protons by an Unbent Half-Wavelength Silicon Crystal, Phys. Rev. Lett. 108, 014801 (2012).

[110] J. E. DeGroote, A. E. Marino, J. P. Wilson, A. L. Bishop, J. C. Lambropoulos, and S. D. Jacobs, Removal rate model for magnetorheological finishing of glass, Appl. Opt. 46, 7927 (2007).

[111] J. A. Menapace, Developing magnetorheological finishing (MRF) technology for the manufacture of large-aperture optics in megajoule class laser systems, in Laser-Induced Damage in Optical Materials: 2010, edited by G. J. Exarhos, V. E. Gruzdev, J. A. Menapace, D. Ristau, and M. J. Soileau (2010). 
[112] A. Authier, Dynamical Theory of X-ray Diffraction (Oxford University Press, Oxford, 2001).

[113] M. Schuster and H. Gobel, Parallel-beam coupling into channel-cut monochromators using curved graded multilayers, J. Phys. D Appl. Phys. 28, A270 (1995).

[114] R. G. Jasinevicius and P. S. Pizani, Annealing treatment of amorphous silicon generated by single point diamond turning, Int. J. Adv. Manuf. Technol. 34, 680 (2007).

[115] A. Kailer, K. G. Nickel, and Y. G. Gogotsi, Raman microspectroscopy of nanocrystalline and amorphous phases in hardness indentations, J. Raman Spectrosc. 30, 939-U7 (1999).

[116] M. A. Green and M. J. Keevers, Optical-Properties of intrinsic silicon at 300 K, Progr. Photovoltaics 3, 189 (1995).

[117] I. Stensgaard, L. C. Feldman, and P. J. Silverman, Calculation of the backscattering-channeling surface peak, Surf. Sci. 77, 513 (1978).

[118] R. L. Kauffman, L. C. Feldman, P. J. Silverman, and R. A. Zuhr, Significance of the channeling surface peak in thin-film analysis, Appl. Phys. Lett. 32, 93 (1978).

[119] V. Baglin, The LHC vacuum system: Commissioning up to nominal luminosity, Vacuum 138, 112 (2017).

[120] P. Chiggiato, J. Somoza, and G. Bregliozzi, Criteria for Vacuum Acceptance Tests (CERN, Geneva, 2018).

[121] M. Butcher, A. Giustiniani, R. Losito, and A. Masi, Controller design and verification for a rotational piezo-based actuator for accurate positioning applications in noisy environments, in IECON 2015-41st Annual Conference of the IEEE Industrial Electronics Society (IEEE, New York, 2015), p. 3887.

[122] A. R. Machado and J. Wallbank, Machining of titanium and its alloys-A review, J. Eng. Manuf. 204, 53 (1990).

[123] H. Akbari, X. Altuna, S. Bardin, R. Bellazzini, V. Biryukov, A. Brez, M. P. Bussa, L. Busso, A. Calcaterra, G. Carboni, F. Costantini, R. De Sangro, K. Elsener, F. Ferioli, A. Ferrari, G. P. Ferri, F. Ferroni, G. Fidecaro, A. Freund, R. Guinand, M. Gyr, W. Herr, A. Hilaire, B. N. Jensen, J. Klem, L. Lanceri, K. Maier, M. M. Massai, V. Mertens, S. P. Møller, S. Morganti, O. Palamara, S. Peraire, S. Petrera, M. Placidi, R. Santacesaria, W. Scandale, R. Schmidt, A. M. Taratin, F. Tosello, E. Uggerhøj, B. Vettermann, P. F. Vita, G. Vuagnin, E. Weisse, and S. Weisz, First results on proton extraction from the CERN-SPS with a bent crystal, Phys. Lett. B 313, 497 (1993).

[124] W. Scandale, G. Arduini, M. Butcher, F. Cerutti, S. Gilardoni, L. Lari, A. Lechner, R. Losito, A. Masi, A. Mereghetti, E. Metral, D. Mirarchi, S. Montesano, S. Redaelli, P. Schoofs, G. Smirnov, E. Bagli, L. Bandiera, S. Baricordi, P. Dalpiaz, V. Guidi, A. Mazzolari, D. Vincenzi, G. Claps, S. Dabagov, D. Hampai, F. Murtas, G. Cavoto, M. Garattini, F. Iacoangeli, L. Ludovici, R. Santacesaria, P. Valente, F. Galluccio, A. G. Afonin, M. K. Bulgakov, Y. A. Chesnokov, V. A. Maisheev, I. A. Yazynin, A. D. Kovalenko, A. M. Taratin, V. V. Uzhinskiy, Y. A. Gavrikov, Y. M. Ivanov, L. P. Lapina, W. Ferguson, J. Fulcher, G. Hall, M. Pesaresi, M. Raymond, and V. Previtali,
Optimization of the crystal assisted collimation of the SPS beam, Phys. Lett. B 726, 182 (2013).

[125] D. De Salvador, E. Bagli, O. Lytovchenko, A. Mazzolari, S. Carturan, G. D Mea, V. Guidi, M. Bazzan, N. Argiolas, A. Carnera, D. Bolognini, S. Hasan, M. Prest, and E. Vallazza, Steering of an ultrarelativistic proton beam by a bent germanium crystal, Appl. Phys. Lett. 98, 234102 (2011).

[126] W. Scandale, I. Efthymiopoulos, D. A. Still, A. Carnera, G. Della Mea, D. De Salvador, R. Milan, A. Vomiero, S. Baricordi, S. Chiozzi, P. Dalpiaz, C. Damiani, M. Fiorini, V. Guidi, G. Martinelli, A. Mazzolari, E. Milan, G. Ambrosi, P. Azzarello, R. Battiston, B. Bertucci, W. J. Burger, M. Ionica, P. Zuccon, G. Cavoto, R. Santacesaria, P. Valente, E. Vallazza, A. G. Afonin, V. T. Baranov, Y. A. Chesnokov, V. I. Kotov, V. A. Maisheev, I. A. Yazynin, S. V. Afanasiev, A. D. Kovalenko, A. M. Taratin, N. F. Bondar, A. S. Denisov, Y. A. Gavrikov, Y. M. Ivanov, V. G. Ivochkin, S. V. Kosyanenko, L. P. Lapina, P. M. Levtchenko, A. A. Petrunin, V. V. Skorobogatov, V. M. Suvorov, D. Bolognini, L. Foggetta, S. Hasan, and M. Prest, Apparatus to study crystal channeling and volume reflection phenomena at the SPS H8 beamline, Rev. Sci. Instrum. 79, 023303 (2008).

[127] E. Bagli, L. Bandiera, V. Guidi, A. Mazzolari, D. De Salvador, A. Berra, D. Lietti, M. Prest, and E. Vallazza, Steering efficiency of a ultrarelativistic proton beam in a thin bent crystal, Eur. Phys. J. C 74, 2740 (2014).

[128] V. Guidi, L. Lanzoni, and A. Mazzolari, Study of anticlastic deformation in a silicon crystal for channeling experiments, J. Appl. Phys. 107, 113534 (2010).

[129] E. Bagli, S. Baricordi, P. Dalpiaz, V. Guidi, A. Mazzolari, D. Vincenzi, A. Carnera, D. D. Salvador, G. D. Mea, and A. M. Taratin, Fabrication of Silicon Strip Crystals for UA9 Experiment (IPCA'10, Kyoto, 2010).

[130] R. Rossi, G. Cavoto, D. Mirarchi, S. Redaelli, and W. Scandale, Measurements of coherent interactions of $400 \mathrm{GeV}$ protons in silicon bent crystals, Nucl. Instrum. Methods Phys. Res. Sect. B 355, 369 (2015).

[131] P. Schoofs, Monte Carlo modeling of crystal channeling at high energies, in Faculté des Sciences de Base, Laboratoire LPAP (École Polytechnique Fédérale de Lausanne, Lausanne, 2013).

[132] F. Andrisani, Selection criteria of silicon bent crystals for beam collimation in the LHC, inScuola di Ingegneria Industriale e dell'Informazione (Politecnico Milano, Milano, 2015).

[133] R. Rossi, Experimental assessment of crystal collimation at the large hadron collider, in Department of Physics (Sapienza Università di Roma, Rome, 2018).

[134] V. Zhovkovska, Characterisation of the Bent Silicon Crystals and Study of the Inelastic Nuclear Interactions of $180 \mathrm{GeV/c}$ Pions in Bent Crystals at the UA9 Experiment (CERN, Geneva, 2018).

[135] Vacuum Acceptance Tests for LHC Collimators, 2011: CERN EDMS document 1113402. 